\title{
Covariance-Based Uncertainty Analysis of Reference Equations of State
}

\author{
Cheung, Howard; Frutiger, Jerome; Bell, Ian H.; Abildskov, Jens; Sin, Gurkan; Wang, Shengwei
}

Published in:

Journal of Chemical and Engineering Data

Link to article, DOI:

10.1021/acs.jced.9b00689

Publication date:

2020

Document Version

Peer reviewed version

Link back to DTU Orbit

Citation (APA):

Cheung, H., Frutiger, J., Bell, I. H., Abildskov, J., Sin, G., \& Wang, S. (2020). Covariance-Based Uncertainty Analysis of Reference Equations of State. Journal of Chemical and Engineering Data, 65(2), 503-522. https://doi.org/10.1021/acs.jced.9b00689

\section{General rights}

Copyright and moral rights for the publications made accessible in the public portal are retained by the authors and/or other copyright owners and it is a condition of accessing publications that users recognise and abide by the legal requirements associated with these rights.

- Users may download and print one copy of any publication from the public portal for the purpose of private study or research.

- You may not further distribute the material or use it for any profit-making activity or commercial gain

- You may freely distribute the URL identifying the publication in the public portal 


\title{
Covariance-based uncertainty analysis of reference equations of state
}

\author{
Howard Cheung, ${ }^{*, \dagger}$ Jérôme Frutiger, ${ }^{*, \ddagger}$ Ian H. Bell, ${ }^{*} \uparrow$ Jens Abildskov, ${ }^{\ddagger}$ Gürkan \\ Sin, ${ }^{\ddagger}$ and Shengwei Wang ${ }^{\S}$ \\ †Carbon Exchange (Hong Kong) Ltd., Shatin, Hong Kong \\ $\ddagger$ Process and Systems Engineering Center (PROSYS), Department of Chemical and \\ Biochemical Engineering, Technical University of Denmark (DTU), 2800 Kgs. Lyngby, \\ Denmark \\ \Applied Chemicals and Materials Division, National Institute of Standards and \\ Technology, Boulder, CO, 80305, USA \\ $\S$ Department of Building Services Engineering, The Hong Kong Polytechnic University, \\ Hung Hom, Hong Kong
}

E-mail: howard.cheung@carbonexchange.net; jfru@kt.dtu.dk; ian.bell@nist.gov

\section{Abstract}

This work presents a detailed methodology for uncertainty analysis applied to a reference equation of state (EOS) based on Helmholtz energy. With increasing interest in uncertainties of thermal process models, it is important to quantify the property uncertainties from the EOS. However, literature relating to EOS development and parameter estimation either does not report uncertainties or report underestimated values. This work addresses the issue by introducing a covariance-based methodology of uncertainty analysis based on a linear approximation. The uncertainty ranges of the EOS properties (95\% confidence intervals) are calculated from the experimental values and the EOS model structure through the parameter covariance matrix and subsequent linear error propagation. In this case study, the Helmholtzbased EOS of propane is analyzed.The uncer- ${ }^{12}$ tainty methodology is general and is applicable ${ }^{13}$ to any novel or existing EOS because it does ${ }^{14}$ not re-train the EOS. The study demonstrates ${ }^{15}$ the insights a thorough uncertainty analysis can ${ }^{16}$ give for EOS users and developers. Uncertain- ${ }^{17}$ ties vary strongly as a function of the state ${ }^{18}$ point, and uncertainties of saturation properties are much larger than the uncertainties of the vapor region due to the use of Maxwell criteria to calculate the saturation properties.

Keywords. Uncertainty analysis, Equation of state, Thermodynamic property, Covariance

\section{Introduction}

2 In recent years, there have been several applications of uncertainty analysis of thermal systems. This includes the selection of working fluids for Rankine cycles, ${ }^{1]}$ virtual sensors of air conditioning systems,,$\frac{213}{}$ chiller control systems, ${ }^{\frac{14}{4}}$ and evaluation of chiller performance models for fault detection and diagnostics (FDD) algorithms. ${ }^{5}$ There have been systematic efforts in describing the uncertainty of both fundamental physicochemical models ${ }^{\sqrt{6}}$ as well as correlation-based property models for process engineering applications. ${ }^{7 / 8}$ The uncertainties of equations of state (EOS) have not yet been adequately studied; most studies neglect the impact of the uncertainty of the EOS. On the other hand, the accuracy of the EOS is commonly considered in the literature, but analy- 
ses of accuracy only consider the difference be- 68 tween the output predicted by the model and 69 experimental data. The consideration of accu- 70 racy instead of uncertainty is common among 71 studies of EOS of different substances. ${ }^{9 \sqrt{12}}$ To 72 this extent, the accuracy is different from the 73 uncertainty of the output of the EOS, which 74 is the range of statistically possible outcomes 75 of the model given different property observa- 76 tions and is usually reported by the $95 \%$ con- 77 fidence interval. Uncertainty of the EOS may 78 also be as important as the other uncertainties. 79 For example, the accuracy of the curve fit for 80 the saturation pressure for the refrigerant blend 81 $\mathrm{R} 410 \mathrm{~A}^{13}$ is $0.5 \%$ of the pressure value, and this 82 is on par with the measurement uncertainty of 83 refrigerant pressure. ${ }^{14}$ It is crucial, in the scope 84 of good modeling practices, to take the uncer- 85 tainties of EOS into account in order to estab- 86 lish the application range and the reliability of ${ }_{87}$ a thermal systems model.

In this manuscript a clear distinction is made 89 between accuracy and uncertainty. The authors 90 are aware that some studies ${ }^{13}$ also consider un- 91 certainty to be the difference between modeled 92 and measured data, and do not use the term 93 accuracy. However, for the sake of clarity, we 94 intend to use the term accuracy and uncertainty 95 separately in this manuscript.

Accuracy and uncertainty are different mea- 97 sures and are both important to assess the per- 98 formance of a model. $\frac{15 \mid 16}{16}$ Analysis of model ac- 99 curacy in the literature of multiparameter ref- 100 erence EOS has mostly so far only involved 101 the calculation of the closeness between some 102 model results and known measurement val- 103 ues. ${ }^{12}$ While a comparison of "closeness" can 104 validate if a model yields an accurate repre- 105 sentation of measurement values, it cannot de- 106 scribe the overall reliability of model results, ${ }_{107}$ and this model accuracy analysis does not tell 108 the whole story for property estimates that are 109 not associated with any known measurement 110 values. The effect of the lack of information 111 can only be quantified by uncertainty quantifi- 112 cation. 17 Some variables calculated by the EOS 113 such as enthalpy and entropy can only be mea- 114 sured through differences relative to a reference 115 point, and it is critical to conduct uncertainty 116 calculations for the estimates of these variables by approaches such as covariance matrix calculation to show the variability of the estimated enthalpy and entropy differences. Furthermore, uncertainty propagation also helps a user to understand how the uncertainty of an EOS affects the result of a thermodynamic cycle model or other process models, especially, when the operation characteristics of the cycle cannot be measured and hence an analysis of model accuracy is unavailable. To do so, it is necessary to make an analysis of the uncertainty of the EOS in addition to the commonly performed analyses on their accuracy.

Some studies ${ }^{14 \mid 18}$ consider the accuracy of EOS of refrigerants ${ }^{9} 12$ as the uncertainties of EOS, and they ignore the effect of the development of the EOS to the uncertainties of EOS. Two studies were conducted to account for the uncertainty due to the covariance. $\frac{1920}{}$ Both of them propagated the uncertainty of measurement data that were used to fit the EOS for the uncertainty of the EOS. One of them required complete knowledge of the uncertainty of measurement data to build the EOS which is unavailable for some EOS. 19 The other one only involved covariance between the input data and the EOS and did not involve covariance between the EOS parameters - a crucial part of EOS uncertainty. ${ }^{20}$ In addition, the method ${ }^{20}$ required re-fitting of the EOS which would be too difficult to be used for all existing EOS. Other studies were conducted to show how to compare the propagation of the EOS parameter uncertainties to the output of a process model. The uncertainties of two EOS (cubictype and SAFT-type) were analyzed. Two of the methods did not use the covariance matrix, but used a bootstrap method as an alternative to estimate the uncertainties of the EOS parameters. ${ }^{21|22|}$ While the methods performed well for specific applications, it was too computationally expensive for general use. There is a need to find another method that is less computationally expensive to quantify the uncertainty of EOS of fluids. Lasala et al. ${ }^{23}$ presented a methodology to quantify and propagate the uncertainty of cubic equations of state as well-as residual Helmholtz energy-based mixing rules 
for fluids used in CO2 Capture processes using the covarianced-based method. However, this method has not been applied to Helmholtzbased equations of state for pure components with large number of parameters.

There is also an increasing industrial interest in the analysis of the uncertainty of EOS and the state-of-the-art is to conduct the uncertainty analysis using only the accuracy of the EOS in the literature ${ }^{12}$ or the propagation of the uncertainties from the inputs ${ }^{24}$ for computationally efficient calculation. However, these two techniques are insufficient to cover important uncertainty components in the estimation of a thermodynamic property of a pure substance as shown in Table 1 . 


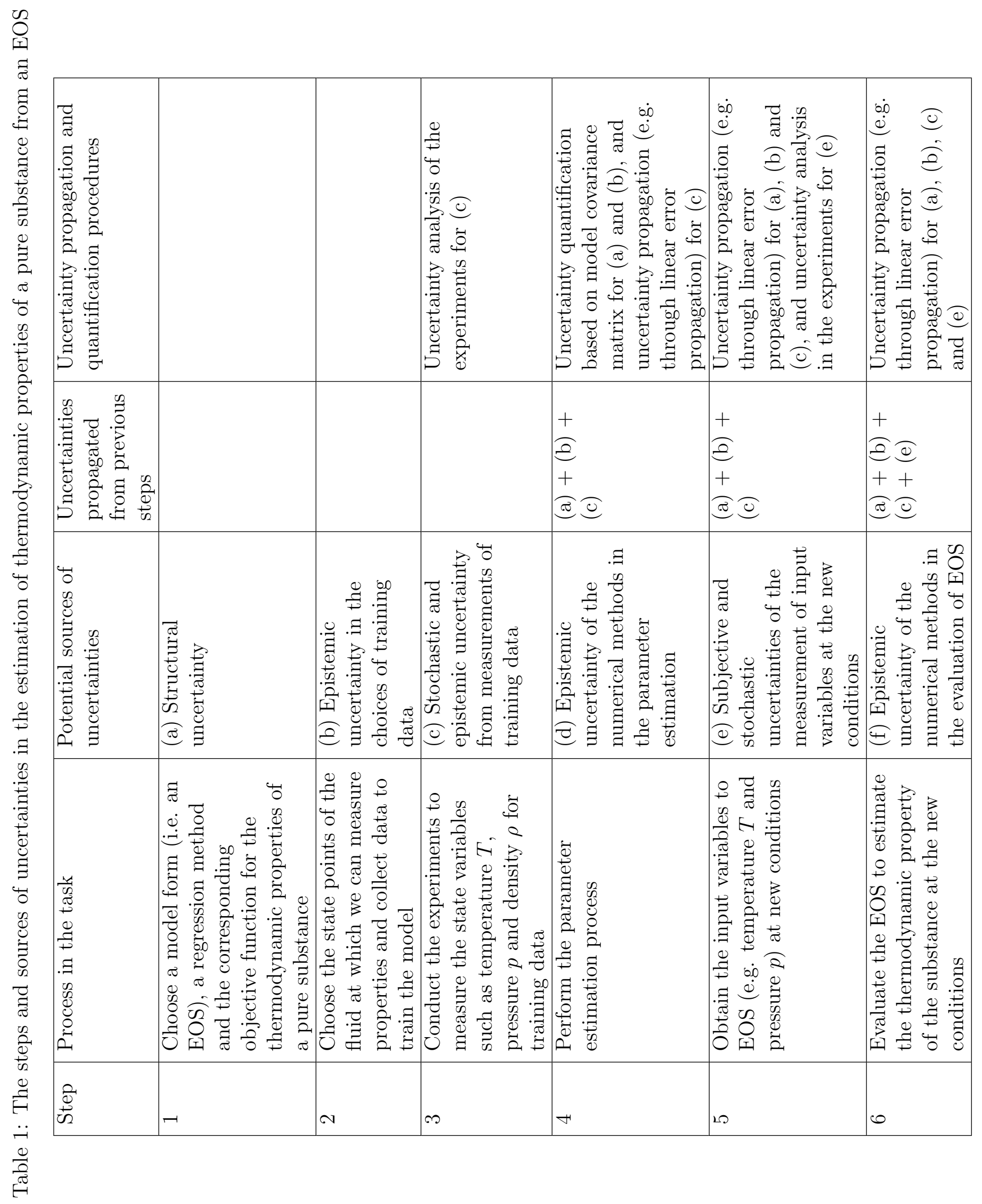


Table 1 shows the sources of uncertainties in 182 the various steps to build an EOS of a pure 183 substance and to use the EOS to estimate a 184 thermodynamic property of the substance. It 185 categorizes the uncertainty sources by struc- 186 tural uncertainties, epistemic (systematic) un- 187 certainties, and stochastic uncertainties accord- 188 ing to Sin et al. ${ }^{[25}$ In step 1, there are structural 189 uncertainties for the mathematical form of the 190 EOS, because other mathematical forms could 191 be used to construct the EOS. Step 2 adds epis- 192 temic uncertainties to the process because the 193 choices of training data of the EOS are subjec- 194 tive. Step 3 adds the epistemic and stochastic 195 uncertainties of experimental setups and sen- 196 sors, and different training data points have ${ }_{197}$ different uncertainty values. ${ }^{26}$ Step 4 only in- 198 troduces epistemic uncertainties of the numer- 199 ical errors of numerical methods in the param- 200 eter estimation. The uncertainties involved are 201 rather typically small (less than $10^{-6} \%$ of the 202 estimated dependent variable) $)^{18}$ and are not 203 calculated. However, the same process also en- 204 ables the calculation of uncertainties of param- 205 eters which are highly related to the uncertain- 206 ties in Steps 1 and 2. Step 5 is similar to Step 3: ${ }_{207}$ another set of data from experiments for the in- 208 puts to the EOS is considered that involves the 209 epistemic and stochastic uncertainties of new 210 measurement values. Step 6 is the use of the ${ }_{211}$ EOS and may involve numerical iterations to 212 solve implicit equations, and hence its uncer- 213 tainties are not calculated.

Table 1 not only shows the sources of uncer- 215 tainties but also a critical feature of the uncer- 216 tainty of a model: the uncertainty of a model ${ }_{217}$ output at a condition is not only determined 218 by the uncertainty of training data at that con- 219 dition. While intuition suggests that the un- 220 certainty of a model at an accurately measured ${ }_{221}$ training data point should be small, the uncer- 222 tainty of a model output at the data point also 223 depends on other factors such as the structural ${ }_{224}$ uncertainty and the subjective uncertainties in 225 the choices of training data. For example, if the 226 structure of the model propagates the large un- 227 certainty of model outputs at some conditions 228 to model outputs at other conditions, the un- 229 certainty of model outputs at other conditions 230 may become larger than expected even if the corresponding training data points are observed accurately. Hence factors that affect the uncertainties of models in addition to measurement uncertainties of model inputs must be considered.

While there are many important components of uncertainties as shown in Table 1, previously reported methods only cover part of them. The "uncertainties" mentioned in the work of Lemmon et al. ${ }^{12}$ only partially involve the calculation of the sum of squares of errors between estimation and measurement values of thermodynamic properties and part of the uncertainty components (a) and (b) in Table 1. Uncertainties propagated by the method of Kline and McClintock ${ }^{24}$ only involve uncertainty component (e). Some methods also fail to address issues such as difficulty to refit the EOS and lack of knowledge of measurement uncertianties of the training data. Hence a study should be conducted to perform the uncertainty analysis of EOSs with other methods.

This paper aims to describe a covariancebased method to calculate uncertainty due to both the sum of squares of residuals and the covariance matrix of the EOS of fluids with the same applicable range as the EOS, using the example of a multiparameter reference EOS of propane (CAS registry number: 74-98-6). 12 The developed method does not require refitting of the equation of state of the fluid and can be used for an EOS of any pure substance. The method is written in a general form. If full and reliable measurement data are available, the methodology allows to take measurement uncertainty into account. However, if comprehensive information of the uncertainty of the training data is not available, the methodology shows how the EOS prediction uncertainty can be calculated without measurement uncertainty. Hence, the method invites both developers and users of EOS to consider the uncertainty through the covariance.

To validate the method, study its applicability, and illustrate its applications, the uncertainty is visualized in the saturation dome of the temperature-entropy and pressure-enthalpy diagrams. The uncertainties of sound speed, 
specific heat, and density at different temper- 274 ature and pressure are also visualized to study 275 how they change under various conditions.

\section{Uncertainty of parame- ${ }_{279}^{278}$ ter estimation by param- ${ }^{280}$ eter covariance matrix}

In this paper, the uncertainty calculation presented is based on the parameter covariance ${ }_{285}$ matrix. .2728 The parameter covariance matrix ${ }_{286}^{286}$ quantifies the uncertainties of the parameters in an equation from a linear regression. It is calculated based on a linear approximation to a regression problem - the commonly performed ${ }^{287}$ regression for the fitting of the EOS parameters ${ }^{288}$ to experimental values - and is helpful for the 289 derivation of the uncertainty of output of the 290 regression equation - the equation of state. 291

As an example, we consider a property $y$ that 292 is described by a model $F(\mathbf{X}, \theta)$, with $\mathbf{X}$ as the ${ }^{293}$ matrix of input variables and $\theta$ being a vec- ${ }^{294}$ tor of model parameters. ${ }^{29} \mathbf{X}$ represents the ${ }^{295}$ experimental measurements. The uncertainty analysis is performed after the successful identification of the best parameter estimates $\theta^{*}$ through non-linear regression (fitting to experimental values by minimization of an objective function) and is begun by the calculation of its parameter covariance matrix.

The calculation of the parameter covariance matrix allows linear error propagation for nonlinear regression models. Considering the above-mentioned property $y$ described 296 by $F(\mathbf{X}, \theta)$, the underlying assumption of this 297 method for uncertainty analysis is that the er- 298 rors $\epsilon$ (i.e. the differences between the model 299 prediction and the experiments) are indepen- 300 dently distributed and defined by a Gaussian 301 distribution white noise (normal distribution 302 with zero mean and unit standard deviation $\sigma$ ) ${ }_{303}$ as expressed by Eq. (1).

$$
y=F(\mathbf{X}, \theta)+\epsilon \quad \epsilon \sim N\left(0, \sigma^{2}\right)
$$

where $y$ is a dependent variable of a regression ${ }^{307}$ equation, $\mathbf{X}$ is a matrix of independent variable ${ }^{308}$ of a regression equation, $F$ is a function of $\mathrm{a}^{309}$

regression equation, $\theta$ is a parameter vector of a regression equation, $\epsilon$ is an error of a regression equation, $\sigma$ is a standard deviation, $N\left(0, \sigma^{2}\right)$ is a Normal distribution with mean at zero and standard deviation at $\sigma$.

To calculate the parameter covariance matrix from the results of Eq. (11), other terms such as the sum of squared errors and the Jacobian of function $F(\mathbf{X}, \theta)$ in Eq. (1) are needed. From the parameter estimation, the weighted sum of squared errors $S S E$ between the experimental and predicted data ( $y^{\exp }$ and $F(\mathbf{X}, \theta)$ ) can be quantified as Eq. (2).

$$
S S E=\Sigma_{i} w_{i} \cdot\left(y_{i}^{\exp }-F(\theta)\right)^{2}
$$

where $S S E$ is the sum of squares of errors, exp means experimental data, and $w_{i}$ is the weighting factor in multi-variable non-linear regression

The Jacobian of function $F(\mathbf{X}, \theta)$ represents the local sensitivity of the property model $F(\mathbf{X}, \theta)$ with respect to the parameter values $\theta$. It can be calculated by taking partial derivatives of $F(\mathbf{X}, \theta)$ as shown in Eq. (3).

$$
J(\mathbf{X}, \theta)=\left[\begin{array}{ccccc}
\frac{\partial F\left(x_{1}, \theta\right)}{\partial \theta_{1}} & \ldots & \frac{\partial F\left(x_{1}, \theta\right)}{\partial \theta_{k}} & \ldots & \frac{\partial F\left(x_{1}, \theta\right)}{\partial \theta_{m}} \\
\ldots & \ldots & \ldots & \ldots & \ldots \\
\frac{\partial F\left(x_{i}, \theta\right)}{\partial \theta_{k}} & \ldots & \frac{\partial F\left(x_{i}, \theta\right)}{\partial \theta_{k}} & \ldots & \frac{\partial F\left(x_{i}, \theta\right)}{\partial \theta_{m}} \\
\ldots & \ldots & \ldots & \ldots & \ldots \\
\frac{\partial F\left(x_{n}, \theta\right)}{\partial \theta_{1}} & \ldots & \frac{\partial F\left(x_{n}, \theta\right)}{\partial \theta_{k}} & \ldots & \frac{\partial F\left(x_{n}, \theta\right)}{\partial \theta_{m}}
\end{array}\right]
$$

Its adaptation and calculation steps for EOS will be discussed in later sections in detail. The parameter $w_{i}$ is the weighting factor of the residuals $\left(y_{i}^{\exp }-F(\theta)\right)$. When there is no weighting needed, $w_{i}$ is assigned to be 1 . In multivariate non-linear regression, ${ }^{27}$ when fitting the experimental data of different properties and orders of magnitude (e.g., pressure and temperature), a weighting factor is needed to normalize the residuals, and $w_{i}$ will be set to values such as $1 /\left(y_{i}^{\exp }\right)^{2}$.

The covariance matrix of the parameters is in its general form written as a function of the Jacobian matrix, $J\left(\mathbf{X}, \theta^{*}\right)$, and the measurement 


$$
\operatorname{COV}\left(\theta^{*}\right)=\left(J\left(\mathbf{X}, \theta^{*}\right)^{\mathrm{T}} \cdot V^{-1} \cdot J\left(\mathbf{X}, \theta^{*}\right)\right)^{-1}
$$

$J\left(\mathbf{X}, \theta^{*}\right)$ expresses the derivatives of $F(\mathbf{X}, \theta){ }^{323}$ with respect to all parameters and is evaluated ${ }_{325}^{324}$ at parameter estimates $\theta^{*}$. $V$ is a matrix con- ${ }_{326}$ taining the measurement uncertainty for each ${ }_{327}$ data point as diagonal elements. Furthermore, ${ }_{328}$ correlation information between the respective measurement values can be represented as offdiagonal elements. The elements of $V$ are represented as variances $\sigma^{2}$, where $\sigma_{k, k}$ would represent the standard deviation of measurement 329 $k$ and $n$ the number of measured data points: 330

$$
V=\left[\begin{array}{ccccc}
\sigma_{1,1}^{2} & \ldots & \sigma_{1, k}^{2} & \ldots & \sigma_{1, n}^{2} \\
\ldots & \ldots & \ldots & \ldots & \ldots \\
\sigma_{1, k}^{2} & \ldots & \sigma_{i, k}^{2} & \ldots & \sigma_{k, n}^{2} \\
\ldots & \ldots & \ldots & \ldots & \ldots \\
\sigma_{n, 1}^{2} & \ldots & \sigma_{n, k}^{2} & \ldots & \sigma_{n, n}^{2}
\end{array}\right]
$$

If accurate measurement uncertainties are ${ }^{337}$ available, Eq- (5) can be used directly. How ever, correlation information is often not avail- ${ }_{340}^{339}$ able for the measurement values or it is assumed that the measurements were uncorrelated. Hence, $V$ is approximated as a diagonal matrix with measurement uncertainties:

$$
\begin{aligned}
V & =\left[\begin{array}{ccccc}
\sigma_{1,1}^{2} & 0 & 0 & 0 & 0 \\
0 & \ldots & 0 & 0 & 0 \\
0 & 0 & \sigma_{k, k}^{2} & 0 & 0 \\
0 & 0 & 0 & \ldots & 0 \\
0 & 0 & 0 & 0 & \sigma_{n, n}^{2}
\end{array}\right] \\
V & =\operatorname{diag}\left[\sigma_{1,1}^{2}, \ldots, \sigma_{k, k}^{2}, \ldots, \sigma_{n, n}^{2}\right]
\end{aligned}
$$

(6) 342 343 344 345 tio

$x$ tim method, $n$ is the number of data points, and $m$ is the number of parameters.

Hence, the covariance matrix of parameters can be re-written by Eq. (9). 27

$$
\operatorname{COV}\left(\theta^{*}\right)=\sigma^{2}\left(J\left(\mathbf{X}, \theta^{*}\right)^{\mathrm{T}} J\left(\mathbf{X}, \theta^{*}\right)\right)^{-1}
$$

where $C O V$ is a covariance matrix, $\theta^{*}$ is an estimated parameter vector of a regression equation and $J$ is a Jacobian matrix.

In Eq. (9), $S S E$ is the value of the sum of squared errors objective function obtained from the least-squares parameter estimation method, $n$ is the number of data points, and $m$ is the number of parameters.

With $\operatorname{COV}\left(\theta^{*}\right)$, the interdependence of the parameters in the property model $F(\mathbf{X}, \theta)$ can be quantified. This is done by calculating the corresponding elements of the parameter correlation matrix obtained by Eq. (10).

$$
\operatorname{Corr}\left(\theta_{i}^{*}, \theta_{j}^{*}\right)=\frac{\operatorname{COV}\left(\theta_{i}^{*}, \theta_{j}^{*}\right)}{\sqrt{\operatorname{Var}\left(\theta_{i}^{*}\right) \operatorname{Var}\left(\theta_{j}^{*}\right)}}
$$

where Corr means correlated coefficient and Var is a Variance

In Eq. $10 p, \operatorname{COV}\left(\theta_{i}^{*}, \theta_{j}^{*}\right)$ is the respective element of the covariance matrix, and $\operatorname{Var}\left(\theta_{i}^{*}\right)$ and $\operatorname{Var}\left(\theta_{j}^{*}\right)$ are the variances of the respective parameters.

The covariance matrix of the property model predictions can be approximated by linear error propagation through the Jacobian of a vector of independent variables $\vec{x}$ and the covariance of the parameter estimates as shown in Eq. (11).

$$
C O V\left(y^{\text {pred }}\right)=J\left(\vec{x}, \theta^{*}\right) C O V\left(\theta^{*}\right) J\left(\vec{x}, \theta^{*}\right)^{\mathrm{T}}
$$

$$
\sigma^{2} \approx \frac{S S E}{n-m}
$$

where pred means predicted values. With the covariance matrices of the param- 
eters and the property model predictions, the 385 uncertainty of the parameters and the property 386 model predictions can be quantified using the 387 confidence intervals of the parameters and prop- 388 erty model predictions. If the assumptions be- 389 hind the model are satisfied (as assumed in the 390 previous steps), the parameter estimates will 391 follow a Student $t$-distribution and the confi- 392 dence interval of parameters can be expressed 393 as Eq. (12).

$\theta_{1-\gamma_{\mathrm{t}} / 2}^{*}=\theta^{*} \pm \sqrt{\operatorname{diag}\left(\operatorname{COV}\left(\theta^{*}\right)\right)} \cdot t\left(n-m, \gamma_{\mathrm{t}} / 2\right)_{396}^{395}$

where $\gamma_{\mathrm{t}}$ is the Student $t$ distribution percentile and diag is a vector formed by the diagonal entries of a matrix.

Similarly, the confidence intervals of the property predictions are given by Eq. (13).

$$
\begin{aligned}
& y_{1-\gamma_{\mathrm{t}} / 2}^{\text {pred }}-y^{\text {pred }} \\
= & \pm \sqrt{\operatorname{diag}\left(C O V\left(y^{\text {pred }}\right)\right)} \cdot t\left(n-m, \gamma_{t} / 2\right)
\end{aligned}
$$

In Eqs. (12) and (13), $t\left(n-m, \gamma_{\mathrm{t}} / 2\right)$ is the Stu- ${ }_{405}^{404}$ dent $t$-distribution value corresponding to the ${ }_{406}$ $\gamma_{\mathrm{t}} / 2$ percentile of the Student $t$-distribution, ${ }_{407}$ $\operatorname{diag}\left(C O V\left(\theta^{*}\right)\right)$ represents the diagonal el- ${ }_{408}^{407}$ ements of $\operatorname{COV}\left(\theta^{*}\right)$, and $\operatorname{diag}\left(\operatorname{COV}\left(y^{\text {pred }}\right)\right)_{409}^{408}$ are the corresponding diagonal elements of ${ }_{410}^{409}$ COV $\left(y^{\text {pred }}\right)$.

The confidence intervals from Eqs. (12) ${ }_{412}^{411}$ and 13 can be regarded as the uncertainty ${ }_{413}$ of the parameters and the property model predictions, respectively, in very simple regression models with uncorrelated parameters. They ${ }^{414}$ quantify the range of possible outcomes of the 415 EOS if the regression process is repeated with data points obtained at other experimental ${ }^{416}$ conditions. However, since all parameters are ${ }^{417}$ correlated with each other to some degree, the basic theory is too simple for complex models like EOS which estimate different types of outputs based upon various types of experimental data. The forthcoming sections describe how the theory can be applied to the EOS to calculate its uncertainty.

The authors would also like to highlight ${ }_{420}^{419}$ the problem of systematic measurement errors. ${ }_{421}$ The assumption of ideally and independently ${ }_{422}$ distributed measurement errors (according to Eq. (1)) would correspond to completely uncorrelated measurement data (white noise). However, it is likely that data points from the same data source will be correlated and systematically higher or lower than another source. These systematic errors depend on many factors such as the experimental set-up, the location and the experimentalists themselves. In the current methodology these correlated measurement errors have not been systematically incorporated, since it is out of the scope of the current work.

\section{Equation of state and its uncertainty calculation}

The thermodynamic properties of pure fluids were first calculated by using the ideal gas laws and variations of ideal gas laws. $\frac{30[31}{\text { In recent }}$ years, EOS have been developed that are based on the fundamental formulation of the nondimensionalized Helmholtz energy $\alpha=a /(R T)$ with temperature $T$ and density $\rho$ as independent variables. Extensive literature is available on these highly flexible empirical multiparameter equations of state. ${ }^{9[12131}$ The detailed formulations and derivations for the equations of state used in this paper can be found in the supporting material, where all the property equations and variables of the EOS are listed.

\subsection{Uncertainty of properties with $T$ and $\rho$ as inputs}

We use the following formulation of the nondimensionalized Helmholtz energy $\alpha$ with temperature and density as independent variables:

$$
\begin{aligned}
\alpha\left(\left[\theta_{\mathrm{EOS}}, T_{\mathrm{c}}, \rho_{\mathrm{c}}\right], T, \rho\right)= & \alpha^{0}\left(\left[\theta_{\mathrm{EOS}}, T_{\mathrm{c}}, \rho_{\mathrm{c}}\right], T, \rho\right) \\
& +\alpha^{\mathrm{r}}\left(\left[\theta_{\mathrm{EOS}}, T_{\mathrm{c}}, \rho_{\mathrm{c}}\right], T, \rho\right)
\end{aligned}
$$

where $\alpha$ is the dimensionless Helmholtz energy, $\alpha^{0}$ is the ideal gas component of dimensionless Helmholtz energy, $\alpha^{\mathrm{r}}$ is the dimensionless Helmholtz energy due to intermolecular 
forces, $\delta$ the is reduced density, $\tau$ is the recip- ${ }_{469}$ rocal of reduced temperature and EOS means equation of state.

We use the notation $\left[\theta_{\mathrm{EOS}}, T_{\mathrm{c}}, \rho_{\mathrm{c}}\right]$ to clearly indicate that $\theta_{\mathrm{EOS}}, T_{\mathrm{c}}$ and $\rho_{\mathrm{c}}$ are model parameters of the EOS. $\rho_{\mathrm{c}}$ is the density at the critical point and $T_{\mathrm{c}}$ the critical temperature. In some EOS (one example would be that of refrigerant R-134a $\mathrm{a}^{32}$ ), a reference state other than the critical point is used to reduce the temperature and density, but that is not further discussed here. In this work, we consider the EOS of propane of Lemmon et al. ${ }^{12}$ and the EOS parameters $\theta_{\text {EOS }}$ can be obtained from the same work. From ${ }_{470}$ the non-dimensionalized Helmholtz energy EOS ${ }_{471}$ other fluid properties can be derived and cal- ${ }_{472}$ culated given temperature $T$ in $\mathrm{K}$, density $\rho_{473}$ in $\mathrm{mol} / \mathrm{m}^{3}$ and parameters $\left[\theta_{\mathrm{EOS}}, T_{\mathrm{c}}, \rho_{\mathrm{c}}\right]:$ the ${ }_{474}$ pressure $p$ in $\mathrm{Pa}$, the molar internal energy $u$ in ${ }_{475}$ $\mathrm{J} / \mathrm{mol}$, the molar enthalpy $\mathrm{hin} \mathrm{J} / \mathrm{mol}$, the molar ${ }_{476}$ entropy $s$ in $\mathrm{J} / \mathrm{mol}-\mathrm{K}$, the molar Gibbs energy ${ }_{477}$ $g$ in $\mathrm{J} / \mathrm{mol}-\mathrm{K}$, the molar Helmholtz energy $a$ in ${ }_{478}$ $\mathrm{J} / \mathrm{mol}$, the molar isochoric heat capacity $c_{v}$ in ${ }_{479}$ $\mathrm{J} / \mathrm{mol}-\mathrm{K}$, the molar isobaric heat capacity $c_{p}$ in ${ }_{480}$ $\mathrm{J} / \mathrm{mol}-\mathrm{K}$, the speed of sound $w$ in $\mathrm{m} / \mathrm{s}$, the fu- ${ }_{481}$ gacity coefficient $\phi$, the second virial coefficient ${ }_{482}$ $B$ in $\mathrm{m}^{3} / \mathrm{mol}$, the third virial coefficient $C$ in ${ }_{483}$ $\mathrm{m}^{6} / \mathrm{mol}$, and the ideal gas isobaric heat capac- ${ }_{484}$ ity $c_{p 0}$ in $\mathrm{J} / \mathrm{mol}-\mathrm{K} .12 \mid 31$

In the literature, Eq. (14) is conventionally ${ }_{486}$ written in terms of the reduced density $\delta=\rho / \rho_{\mathrm{c}}{ }_{487}$ and reciprocal reduced temperature $\tau=T_{\mathrm{c}} / T{ }_{488}$ where $\delta$ and $\tau$ are the independent variables of ${ }_{489}$ the EOS and $\theta_{\mathrm{EOS}}$ are the parameters. How- ${ }_{490}$ ever, there are usually considerable differences ${ }_{491}$ among investigators in the measurement of the ${ }_{492}$ critical temperature $T_{\mathrm{c}}$ and critical density $\rho_{\mathrm{c}}$ as ${ }_{493}$ shown by Lemmon et al. ${ }^{12}$ This means that it is ${ }_{494}$ necessary to take into account the measurement ${ }_{495}$ uncertainties of the critical point when calculat- ${ }_{496}$ ing the uncertainty of the EOS outputs.

The covariance-based uncertainty analysis ${ }_{498}$ method, outlined in Section 2, is now described ${ }_{499}$ for the Helmholtz-based EOS. Equation (14) 500 can be written analogously to Eq. (1) assum- ${ }_{501}$ ing ideally and independently distributed errors ${ }_{502}$ defined by a Gaussian distribution white noise.

$$
\begin{gathered}
y=F(\mathbf{X}, \vartheta)+\epsilon \quad \epsilon \sim N\left(0, \sigma^{2}\right) \\
\mathbf{y}:=\left[p, u, h, s, g, a, c_{v}, c_{p}, \omega, \phi, B, C, c_{p 0}\right] \\
\mathbf{X}:=(T, \rho) \\
\vartheta:=\left[\theta_{\mathrm{EOS}}, T_{\mathrm{c}}, \rho_{\mathrm{c}}\right]
\end{gathered}
$$

$T_{c}$ and $\rho_{c}$ are defined according to the experimental results of thermodynamic properties around the critical point, and we assume that the parameter estimation for $\theta_{\mathrm{EOS}}$ (parameter optimization and fitting to a large set of experimental data) has been completed by the developers of the EOS. The set of parameters called $\theta_{\mathrm{EOS}}^{*}$ is known from the literature. ${ }^{11}$ Hence, the parameter estimates $\theta_{\mathrm{EOS}}^{*}$ are used in this study for the uncertainty analysis and re-training of the data is not necessary.

The parameters of the EOS are given by $\theta_{\mathrm{EOS}}=\left[\theta_{1}, \ldots, \theta_{j}, \ldots, \theta_{m}\right]$, with $m$ being the number of parameters.

However, experimental data need to be collected or taken from the work of the developers of the EOS because it is needed to calculate the errors (differences between experimental data and predicted values by the EOS). For example, in the case of propane, the EOS has been fitted to experimental data of $p, p_{\text {sat }}, c_{v}, c_{p}, w$, $B, c_{p 0}$ as functions of $T$ and $\rho$. These variables are called fitted (or observed) variables because some of their values are measured and included in the fitting process. Other variables (e.g., $u, h, s, g, a, C$ and $\phi$ ) are called (purely) predicted variables because none of their experimental measurements are included in the fitting process of the EOS. Some EOSs may have different sets of fitted variables and predicted variables depending on experimental data availability, but the proposed method focuses on using the aforementioned set of fitted and predictive variables for simplicity. The parameter covariance matrix (i.e. the uncertainty information of the parameter estimates $\theta_{\mathrm{EOS}}^{*}$ ) is obtained 
through the use of data. The uncertainties of 540 $T, \rho, p, c_{v}, c_{p}, w, B$ and $c_{p 0}$ as well as $u, h, s,{ }_{541}$ $C, \phi, a$ and $g$ estimated by the EOS are then ${ }_{542}$ obtained by linear error propagation.

For the fitted properties, there are a number ${ }_{544}$ of $n$ experimental data points respectively, e.g. 545 $p^{\exp }=\left[p_{1}^{\exp }, \ldots, p_{n_{p}}^{\exp }\right], c_{v}^{\exp }=\left[c_{v 1}^{\exp }, \ldots, c_{v n_{c_{v}}}^{\exp }\right]$, etc. ${ }_{546}$

We describe specifically how the uncertainty ${ }_{547}$ analysis described in Section 2 can be applied to the estimation of the uncertainty of the predictions of EOS outputs. We will use the notation introduced above for $\alpha\left(\left[\theta_{\mathrm{EOS}}, T_{\mathrm{c}}, \rho_{\mathrm{c}}\right], T, \rho\right)$, where the parameters for the EOS are $\theta_{\mathrm{EOS}}, T_{\mathrm{c}}$ and $\rho_{\mathrm{c}}$, and the independent variables are $T$ and $\rho$.

Equation (9) described the covariance matrix ${ }^{548}$ of the parameters determined from the vari- ${ }^{549}$ ances of errors $\sigma^{2}$ and the Jacobian $J\left(\theta^{*}\right)$, where ${ }^{550}$ $\sigma^{2}$ is obtained from the sum of squared errors ${ }_{551}$ $S S E$ of the objective function from the leastsquare parameter estimation. Hence, in the ${ }^{552}$ case of the fundamental EOS for propane, as ${ }^{553}$ described by Lemmon et al., ${ }^{12} S S E$ is described ${ }^{554}$ as the sum of squared relative errors - the dif- 555 ference between the predicted and experimen- ${ }^{556}$ tal property value divided by the experimental ${ }^{557}$ value (least square regression). Hence, for the ${ }^{558}$ example of the fitted property $c_{p}$, its variance ${ }^{559}$ $\sigma^{2}$ should be written as

$$
\sigma_{c_{p}}^{2} \approx \frac{S S E_{c_{p}}}{n_{c_{p}}-m_{c_{p}}}
$$

where $n_{c_{p}}$ is the number of data points for $c_{p}{ }^{564}$ and $m_{c_{p}}$ is the number of EOS parameters that ${ }^{565}$ are needed to calculate $c_{p}$ using the EOS (note ${ }^{566}$ $\left.n_{c_{p}}-m_{c_{p}}>0\right) . S S E_{c_{p}}$ is the value of the sum of ${ }^{567}$ squared relative errors objective function from ${ }^{568}$ the parameter estimation. ${ }^{[12}$ In order to obtain ${ }^{569}$ $S S E_{c_{p}}$ the parameter estimation does not need ${ }^{570}$ to be retrained:

$$
S S E_{c_{p}}=\sum_{i=1}^{n_{c_{p}}}\left[\frac{c_{p_{i}}^{\exp }-c_{p}\left(\theta_{\mathrm{EOS}}^{*}, \tau_{i}, \delta_{i}\right)}{c_{p_{i}}^{\exp }}\right]^{2}
$$

where $i$ is the index for the experimental condition $\left(T_{i}, \rho_{i}\right), c_{p_{i}}^{\exp }$ is the experimental value of specific heat and $c_{p}\left(\theta_{\mathrm{EOS}}^{*}, \tau_{i}, \delta_{i}\right)$ is the predicted ${ }_{574}^{573}$ specific heat value. The estimates for variances of the other fitted properties are obtained for $\sigma_{c_{v}}^{2}, \sigma_{w}^{2}, \sigma_{B}^{2}, \sigma_{c_{p 0}}^{2}$ and $\sigma_{p_{c}}^{2}$. The estimate for the error of $T_{\mathrm{c}}$ and $\rho_{\mathrm{c}}\left(\sigma_{T_{\mathrm{c}}}^{2}\right.$ and $\left.\sigma_{\rho_{\mathrm{c}}}^{2}\right)$ can be calculated from the standard deviation of the experimental measurements. Lemmon et al. ${ }^{12}$ used a different objective function for the residual errors in the pressure as shown in Eq. (21), in order to obtain similar magnitudes of the liquid and vapor phase.

$$
S S E_{p}=\sum_{i}\left[\frac{p_{i}^{\exp }-p\left(\theta_{\mathrm{EOS}}^{*}, \tau_{i}, \delta_{i}\right)}{\rho_{i}^{\exp }\left(\frac{\partial p_{i}}{\partial \rho_{i}}\right)_{T}}\right]^{2}
$$

where $p_{i}^{\exp }$ is the experimental pressure value, $p\left(\theta_{\mathrm{EOS}}^{*}, \tau_{i}, \delta_{i}\right)$ is the predicted pressure value, $\rho_{i}^{\exp }$ is the experimental density value and $\left.\frac{\partial p_{i}}{\partial \rho_{i}}\right|_{T}$ is the partial derivative of the pressure with respect to the density calculated at $\theta_{\mathrm{EOS}}^{*}$.

In order for the uncertainty assessment of the EOS to be consistent with the EOS itself, the exact objective function used in the training of the EOS is needed, but this is very difficult to achieve in practice. The state-of-the-art fitting process includes addition and removal of data points and constraints in an iterative fashion, and it is not possible to obtain the weights that were ultimately used in the regression process. Therefore, the weights that were used in the fitting process of Lemmon et al. ${ }^{12}$ are unknowable, and an estimation of the objective function (i.e. the corresponding SSE) is required.

While Eq. (21) is appropriate for pressure data points with density and temperature as independent variables, it is not suitable for vapor pressure data. For vapor pressure data, the desired residue would be

$$
S S E_{p_{\mathrm{sat}}}=\sum_{i}\left[\frac{p_{i}^{\exp }-p_{\mathrm{sat}}\left(\theta_{\mathrm{EOS}}^{*}, \tau_{i}\right)}{p_{i}^{\exp }}\right]^{2},
$$

though this form is not suitable because the evaluation of $p^{\text {sat }}$ requires an iterative solution for the vapor pressure, which is a calculation fraught with potential perils. For that reason, it is preferrable, and common practice, to mini- 
mize the difference in Gibbs energy between the 614 saturated liquid and vapor phases for the given 615 temperature and pressure (see for instance Bell 616 et al. $\left.{ }^{33}\right)$. In this case, the densities must be 617 solved for in each phase, but the full resolution 618 of the Maxwell criteria is not required.

To describe the minimization process in terms of $S S E$, Eq. (23) is used with vapor pressure data.

$$
\begin{gathered}
S S E_{g}= \\
\sum_{i}\left[\frac{g\left(\theta_{\mathrm{EOS}}^{*}, \tau_{i}, \frac{\rho_{\mathrm{min}, i}}{\rho_{c}}\right)-g\left(\theta_{\mathrm{EOS}}^{*}, \tau_{i}, \frac{\rho_{\mathrm{max}, i}}{\rho_{c}}\right)}{R T_{i}^{\exp }}\right]^{2} \\
\rho_{\min , i}=\min \left(\vec{\rho}\left(T_{i}^{\exp }, p_{i}^{\exp }\right)\right) \\
\rho_{\max , i}=\max \left(\vec{\rho}\left(T_{i}^{\exp }, p_{i}^{\exp }\right)\right)
\end{gathered}
$$

where $\rho_{\min }$ is the minimum density calculated by iteratively solving for density from the specified temperature and pressure, $\rho_{\max }$ is the maximum density calculated from the same iterative calculation of density, $R$ is the universal gas constant, $T_{i}^{\exp }$ is the experimental temperature reading in the vapor pressure data.

One may wonder if the $S S E$ in Eq. (23) is suitable to describe the deviation in pressure differences between estimated and measured vapor pressure. To verify that Eq. (23) is approximately equivalent to Eq. (22), the relative deviation between the estimated and pressure pressure is plotted with the difference of the Gibbs energy of saturated liquid and vapor divided by ${ }_{619}$ the product of the gas constant and $T$, as well ${ }_{620}$ as more accurate approximations to Eq. (22). The derivations in the supplemental material ${ }_{622}$ explain how this non-dimensionalization can be ${ }_{623}$ obtained, following the assumptions that a) ${ }_{624}$ a first-order series expansion of Gibbs energy ${ }_{625}$ with respect to pressure difference can be used, ${ }_{626}$ b) the vapor phase derivative of Gibbs energy ${ }_{627}$ with respect to pressure at constant tempera- ${ }_{628}$ ture is much greater in magnitude than that of ${ }_{629}$ the liquid phase, and c) the vapor phase can be ${ }_{630}$ treated as an ideal gas.

Figure 1 presents numerical values for each ${ }_{632}$ of the approximations to the vapor pressure ${ }_{633}$ residue of propane. This figure demonstrates that $\Delta g /(R T)$ provides a fair representation of the saturation pressure residue although the second-order expansion yields a superior evaluation of the vapor pressure residue.

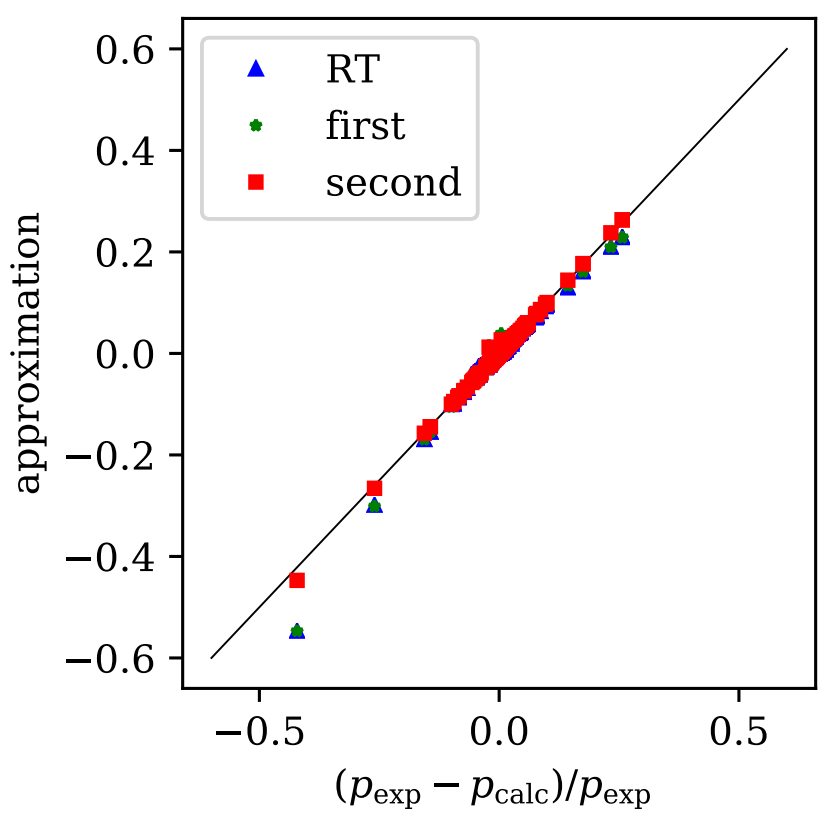

Figure 1: Comparing three different approximations to the deviation in pressure with relative error in pressure calculation of propane. For more information, see the derivations in the supplemental material (RT: res $=\Delta g /(R T)$, first: a first-order expansion in pressure difference, dropping the liquid derivative without any further simplifications, second: a second order expansion in pressure difference without any further simplifications)

The results show that the difference of Gibbs energy can effectively describe the difference between estimated and measured vapor pressure. However, since the differences of pressure are not really used in the estimation of the coefficients in the Helmholtz-energy-based EOS, Eq. (23) is used in this study.

A diagonal matrix containing all the error variances can then be built as follows in Eq. (26).

The $V$ matrix in Eq. (26) contain $2 n_{p_{v}}$ number of $\sigma_{g}^{2}$ because each $\sigma_{g}^{2}$ is calculated by two predicted values: the predicted values of Gibbs energy of saturated vapor and the predicted values of Gibbs energy of saturated liquid. 


$$
V=\operatorname{diag}[\underbrace{\sigma_{p}^{2}, \ldots, \sigma_{p}^{2}}_{n_{p}}, \underbrace{\sigma_{g}^{2}, \ldots, \sigma_{g}^{2}}_{2 n_{p_{v}}}, \underbrace{\sigma_{c_{v}}^{2}, \ldots, \sigma_{c_{v}}^{2}}_{n_{c \mathrm{v}}}, \underbrace{\sigma_{c_{p}}^{2}, \ldots, \sigma_{c_{p}}^{2}}_{n_{c \mathrm{p}}}, \underbrace{\sigma_{w}^{2}, \ldots, \sigma_{w}^{2}}_{n_{w}}, \underbrace{\sigma_{B}^{2}, \ldots, \sigma_{B}^{2}}_{n_{B}}, \underbrace{\sigma_{n_{c_{p 0}}}^{2}, \ldots, \sigma_{n_{c_{p 0}}}^{2}}_{n_{c_{p 0}}}, \underbrace{\sigma_{T_{\mathrm{c}}}^{2}}_{n_{T_{\mathrm{c}}}}, \ldots, \sigma_{T_{\mathrm{c}}}^{2}, \underbrace{\sigma_{\rho_{\mathrm{c}}}^{2}}_{n_{\rho_{\mathrm{c}}}}, \ldots, \sigma_{\rho_{\mathrm{c}}}^{2}]
$$

The Jacobian for all of the fitted properties 677 needs to be calculated at the specific experi- 678 mental measurement point of the variables $T 679$ and $\rho$ (or $\tau$ and $\delta$ respectively for their dimen- 680 sionless form), and at the specific fitted param- 681 eter values $\theta_{\mathrm{EOS}}^{*}$. However, since the variances 682 $\sigma_{p}^{2}, \sigma_{g}^{2}, \sigma_{c_{p}}^{2}, \sigma_{c_{v}}^{2}, \sigma_{w}^{2}, \sigma_{B}^{2}, \sigma_{c_{p 0}}^{2}, \sigma_{T_{\mathrm{c}}}^{2}$ and $\sigma_{p_{\mathrm{c}}}^{2}$ used ${ }_{683}$ relative errors, the elements of the Jacobians 684 need to be normalized by their corresponding 685 experimental value. As an example, the gen- 686 eral analytical expression of the Jacobian of $p{ }_{687}$ can be written as Eq. (27).

While the Jacobians of an experimental data point of most fitted variables can be calculated in a similar way as Eq. (27), the Jacobian of each experimental observation of vapor pressure are calculated differently because they have two predicted values of Gibbs energy in each difference term in Eq. (23). To involve the partial derivatives of both predicted values in the $S S E_{g}$, the Jacobian of vapor pressure is a $2 \times$ $(m+2)$ matrix as Eq. (28)

The first row of the Jacobian in Eq. (28) corresponds to the predicted values of Gibbs energy of the maximum density value at the vapor pressure, and the second row of the Jacobian corresponds to the predicted values of Gibbs energy of the minimum density values at the corresponding temperature.

The Jacobian of the fitted data of $p$ at multiple experimental data points of $\tau$ and $\delta$, evaluated at the parameter estimates $\theta_{\mathrm{EOS}}^{*}$ is then given by Eq. (29), where $J_{p}\left(\theta_{\mathrm{EOS}}^{*}, \tau, \delta\right)$ is a $n_{p} \mathrm{x}(m+2)$ matrix with $\theta_{\mathrm{EOS}}^{*}=\left[\theta_{1}^{*}, \ldots, \theta_{m}^{*}\right]$, $\tau=\left[\tau_{1}, \ldots, \tau_{n_{p}}\right]$ and $\delta=\left[\delta_{1}, \ldots, \delta_{n_{p}}\right] . \quad p_{i}^{\exp }$ are the corresponding experimental values used to normalize the Jacobian. The method to calculate the partial derivatives is discussed in the Supplementary Materials for reference.

Assuming that the uncertainties of critical 689 properties have no effect on the estimation 690 of the coefficients $\theta_{\mathrm{EOS}}$ and do not propagate ${ }_{691}$ through their estimation process, the Jacobian ${ }_{692}$ of $p$ evaluated at the experimental values can be simplified by setting the last two columns of values in Eq. (29) as zeros.

It is important to notice that $J_{p}$ is evaluated at the parameter estimates $\theta_{\mathrm{EOS}}^{*}$, but is a function of $T$ and $\rho$. In analogy, the Jacobian for the other fitted data can be obtained, giving $J_{c_{v}}, J_{c_{p}}, J_{w}, J_{B}$ and $J_{c_{p 0}}$. Since we are accounting for the effect of the uncertainties of critical densities and critical temperature, their Jacobians are given as

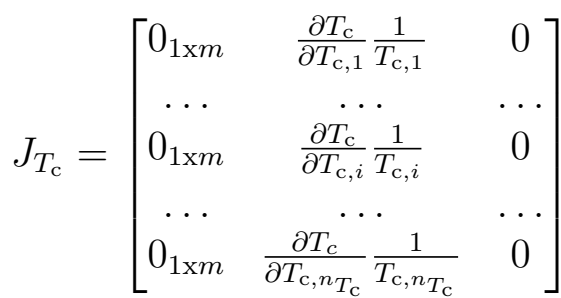

$$
\begin{aligned}
& =\left[\begin{array}{ccc}
0_{1 \times m} & \frac{1}{n_{T_{\mathrm{c}}} T_{\mathrm{c}, 1}} & 0 \\
\ldots & \cdots & \ldots \\
0_{1 \times m} & \frac{1}{n_{T_{\mathrm{c}} T_{\mathrm{c}, i}}} & 0 \\
\ldots & \cdots & \cdots \\
0_{1 \times m} & \frac{1}{n_{T_{\mathrm{c}}} T_{c, n} T_{\mathrm{c}}} & 0
\end{array}\right]
\end{aligned}
$$

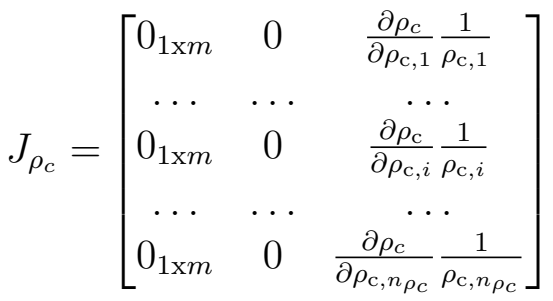

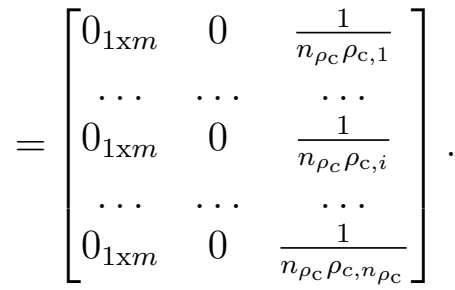

The combined Jacobian of the fitted data $p$, $p_{v}, c_{v}, c_{p}, w, B$ and $c_{p 0}$ can constructed as in Eq. (32).

The Jacobian $J_{\text {tot }}\left(\left[\theta_{\mathrm{EOS}}^{*}, T_{\mathrm{c}}, \rho_{\mathrm{c}}\right], T, \rho, p\right)$ is a $\left(n_{p}+2 n_{p_{v}}+n_{c_{v}}+n_{c_{p}}+n_{w}+n_{B}+n_{c_{p 0}}+n_{T_{\mathrm{c}}}+n_{\rho_{\mathrm{c}}}\right)$ 


$$
J_{p}\left(\left[\theta_{\mathrm{EOS}}^{*}, T_{\mathrm{c}}, \rho_{\mathrm{c}}\right], T_{i}, \rho_{i}\right)=\frac{1}{p_{i}^{\exp }}\left[\frac{\partial p}{\partial \theta_{1}}\left(\theta_{\mathrm{EOS}}^{*}, T_{i}, \rho_{i}\right) \quad \ldots \quad \frac{\partial p}{\partial \theta_{M}}\left(\theta_{\mathrm{EOS}}^{*}, T_{i}, \rho_{i}\right) \quad \frac{\partial p}{\partial T_{\mathrm{c}}}\left(\theta_{\mathrm{EOS}}^{*}, T_{i}, \rho_{i}\right) \quad \frac{\partial p}{\partial \rho_{c}}\left(\theta_{\mathrm{EOS}}^{*}, T_{i}, \rho_{i}\right)\right]
$$

$$
\begin{aligned}
& J_{p_{v}}\left(\left[\theta_{\mathrm{EOS}}^{*}, T_{\mathrm{c}}, \rho_{\mathrm{c}}\right], T_{i}, p_{i}\right)=\frac{1}{R T_{i}} \times \\
& {\left[\begin{array}{lllll}
\frac{\partial g}{\partial \theta_{1}}\left(\theta_{\mathrm{EOS}}^{*}, T_{i}, \rho_{\mathrm{min}, i}\right) & \ldots & \frac{\partial g}{\partial \theta_{M}}\left(\theta_{\mathrm{EOS}}^{*}, T_{i}, \rho_{\mathrm{min}, i}\right) & \frac{\partial g}{\partial T_{\mathrm{c}}}\left(\theta_{\mathrm{EOS}}^{*}, T_{i}, \rho_{\mathrm{min}, i}\right) & \frac{\partial g}{\partial \rho_{c}}\left(\theta_{\mathrm{EOS}}^{*}, T_{i}, \rho_{\min , i}\right) \\
\frac{\partial g}{\partial \theta_{1}}\left(\theta_{\mathrm{EOS}}^{*}, T_{i}, \rho_{\mathrm{max}, i}\right) & \ldots & \frac{\partial g}{\partial \theta_{M}}\left(\theta_{\mathrm{EOS}}^{*}, T_{i}, \rho_{\mathrm{max}, i}\right) & \frac{\partial g}{\partial T_{\mathrm{c}}}\left(\theta_{\mathrm{EOS}}^{*}, T_{i}, \rho_{\mathrm{max}, i}\right) & \frac{\partial g}{\partial \rho_{c}}\left(\theta_{\mathrm{EOS}}^{*}, T_{i}, \rho_{\mathrm{max}, i}\right)
\end{array}\right]}
\end{aligned}
$$

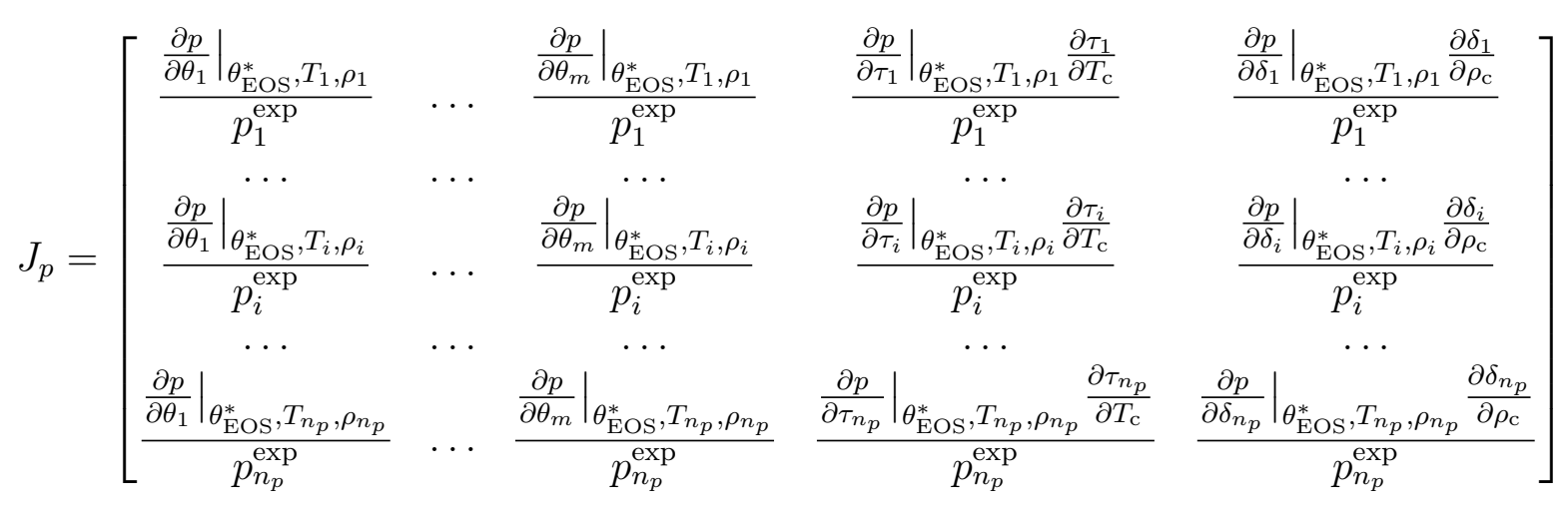

$$
J_{\mathrm{tot}}\left(\left[\theta_{\mathrm{EOS}}^{*}, T_{c}, \rho_{\mathrm{c}}\right], T, \rho, p\right)=\left[\begin{array}{c}
J_{p}\left(\left[\theta_{\mathrm{EOS}}^{*}, T_{\mathrm{c}}, \rho_{\mathrm{c}}\right], T, \rho\right) \\
J_{p_{\mathrm{v}}}\left(\left[\theta_{\mathrm{EOS}}^{*}, T_{\mathrm{c}}, \rho_{\mathrm{c}}\right], T, p\right) \\
J_{c_{v}}\left(\left[\theta_{\mathrm{EOS}}^{*}, T_{\mathrm{c}}, \rho_{\mathrm{c}}\right], T, \rho\right) \\
J_{c_{p}}\left(\left[\theta_{\mathrm{EOS}}^{*}, T_{\mathrm{c}}, \rho_{\mathrm{c}}\right], T, \rho\right) \\
J_{w}\left(\left[\theta_{\mathrm{EOS}}^{*}, T_{c}, \rho_{\mathrm{c}}\right], T, \rho\right) \\
J_{B}\left(\left[\theta_{\mathrm{EOS}}^{*}, T_{c}, \rho_{\mathrm{c}}\right], T\right) \\
J_{c_{p 0}}\left(\left[\theta_{\mathrm{EOS}}^{*}, T_{\mathrm{c}}, \rho_{\mathrm{c}}\right], T\right) \\
J_{T_{\mathrm{c}}} \\
J_{\rho_{\mathrm{c}}}
\end{array}\right]
$$

$\mathrm{x}(m+2)$ matrix. $J_{p_{v}}$ are functions of $T$ and $p 706$ because their $S S E$ in Eq. (23) have no density 707 values as inputs. $J_{B}$ and $J_{c_{p 0}}$ are not functions 708 of $\delta$ because $B$ and $c_{p 0}$ depend on temperature 709 only.

In Eq. (32), with the exception of $J_{p_{v}}$, all 711 output variables are assumed to be associated 712 with measurements of temperature and den- 713 sity. However, in actual experiments, other 714 sets of variables like temperature and pressure 715 are more natural independent variables. Hence, 716 conversion for the measurement data point is 717 needed before it can be used to calculate the 718
Jacobian. For data with temperature and pressure measurement but unknown density values, the density values are calculated from the temperature and pressure measurement via the EOS. The conversion method for density of different variables is outlined in Table 2 .

According to Eq. (9), the covariance matrix can be given as in Eq. (33).

The size of $C O V\left(\left[\theta_{\mathrm{EOS}}^{*}, T_{\mathrm{c}}, \rho_{\mathrm{c}}\right]\right)$ depends on the number of parameters in the EOS. For example, the EOS of propane has 97 parameters that are obtained from measured data in the literature. Taking into account $T_{\mathrm{c}}$ and $\rho_{\mathrm{c}}$, its 


$$
\operatorname{COV}\left(\left[\theta_{E O S}^{*}, T_{\mathrm{c}}, \rho_{\mathrm{c}}\right]\right)=\left[J_{\mathrm{tot}}\left(\left[\theta_{\mathrm{EOS}}^{*}, T_{\mathrm{c}}, \rho_{\mathrm{c}}\right], T, \rho\right)^{\mathrm{T}} \cdot V^{-1} \cdot J_{\mathrm{tot}}\left(\left[\theta_{\mathrm{EOS}}^{*}, T_{\mathrm{c}}, \rho_{\mathrm{c}}\right], T, \rho\right)\right]^{-1}
$$

Table 2: Methods of data conversion for density 735 can be written as values in Jacobian calculation

\begin{tabular}{ll}
\hline \hline $\begin{array}{l}\text { Type of mea- } \\
\text { surement }\end{array}$ & Density values \\
\hline$c_{p 0}$ & Not needed \\
$p, \rho, T$ & From $\rho$ measurement \\
$B$ & only \\
$w, c_{p}$ and $c_{v}$ & $\begin{array}{l}\text { Not needed } \\
\text { Calculated by } T \text { and } p \\
\text { from EOS }\end{array}$ \\
\hline \hline
\end{tabular}

covariance matrix is a $99 \times 99$ square matrix. ${ }^{[12}{ }_{738}^{738}$ The EOS of refrigerant R-22 has 55 parameters ${ }_{740}$ and its covariance matrix is a $57 \times 57$ square ma- ${ }_{741}$ trix. 9

Following Eq. (9), the variance $\sigma_{p}^{2}$ is only mul- ${ }_{743}^{742}$ tiplied with the elements of $J_{p}\left(\theta_{\mathrm{EOS}}^{*}, \tau, \delta\right)$; the ${ }_{744}$ same holds for the other properties. In order to ${ }_{745}$ illustrate this, it is possible to re-write Eq. 32 . ${ }_{746}^{745}$ in the following way

$V^{-1} \cdot J_{\text {tot }}\left(\left[\theta_{\mathrm{EOS}}^{*}, T_{\mathrm{c}}, \rho_{c}\right], T, \rho, p\right)=\left[\begin{array}{c}1 / \sigma_{p}^{2} \cdot J_{p} \\ 1 / \sigma_{g}^{2} \cdot J_{p_{v}} \\ 1 / \sigma_{c_{v}} \cdot J_{c_{v}} \\ 1 / \sigma_{c_{p}}^{2} \cdot J_{c_{p}} \\ 1 / \sigma_{w}^{2} \cdot J_{w} \\ 1 / \sigma_{B}^{2} \cdot J_{B} \\ 1 / \sigma_{c_{p 0}}^{2} \cdot J_{c_{p 0}} \\ 1 / \sigma_{T_{\mathrm{c}}}^{2} \cdot J_{T_{\mathrm{c}}} \\ 1 / \sigma_{\rho_{\mathrm{c}}}^{2} \cdot J_{\rho_{\mathrm{c}}}\end{array}\right]_{750}$ (34) 752

The variances $\sigma$ are essentially weighting fac- 753 tors for the elements of the Jacobian.

To avoid refitting the EOS for the covariance 755 between the coefficients and the critical values, 756 it is assumed that the uncertainties of critical ${ }^{757}$ properties have a negligible effect on the esti- 758 mation of the coefficients $\theta_{\mathrm{EOS}}$. The covariance 759

$$
\begin{gathered}
\operatorname{COV}\left(\left[\theta_{\mathrm{EOS}}^{*}, T_{\mathrm{c}}, \rho_{\mathrm{c}}\right]\right) \\
=\left[\begin{array}{ccc}
\operatorname{COV}\left(\theta_{\mathrm{EOS}}^{*}\right) & 0 & 0 \\
0 & \frac{\sigma_{T_{\mathrm{c}}}^{2}}{T_{\mathrm{c}}^{2}} & 0 \\
0 & 0 & \frac{\sigma_{\rho_{\mathrm{c}}}^{2}}{\rho_{\mathrm{c}}^{2}}
\end{array}\right]
\end{gathered}
$$
(3) C using Eqs. 32 and 33 when $T_{\mathrm{c}}$ and $\rho_{\mathrm{c}}$ are assumed to be known perfectly and have therefore no uncertainty.

Calculating $\operatorname{COV}\left(\theta_{\mathrm{EOS}}^{*}\right)$ based on Eq. (35), it is possible to calculate the respective $95 \%$ confidence interval of the EOS parameter values $\theta_{\mathrm{EOS}}$

$$
\begin{aligned}
& \theta_{\mathrm{EOS}, 1-\gamma_{\mathrm{t}} / 2} \\
= & \left.\theta_{\mathrm{EOS}}^{*} \pm \sqrt{\operatorname{diag}\left(\operatorname{COV}\left(\theta_{\mathrm{EOS}}^{*}\right)\right.}\right) \cdot t\left(n-m, \gamma_{\mathrm{t}} / 2\right)
\end{aligned}
$$

In Eq. (36) $t\left(n-m, \gamma_{\mathrm{t}} / 2\right)$ is the Student $t$ distribution value corresponding to the $\gamma_{t} / 2$ percentile of the Student $t$-distribution.

The covariance of the prediction of a certain property is obtained from the respective Jacobian and the parameter covariance matrix and is dimensionalized so that its unit should be the square of that of the property. For example, the covariance $C O V(p)$ for a pressure $p$ is given by Eq. (37) and the jacobian vector in Eq. (38).

The $C O V\left(\left[\theta_{\mathrm{EOS}}^{*}, T_{\mathrm{c}}, \rho_{\mathrm{c}}\right]\right)$ is a matrix with elements independent of the input variables to EOS. However, the adjusted Jacobian $j_{p}\left(\left[\theta_{\mathrm{EOS}}^{*}, T_{\mathrm{c}}, \rho_{\mathrm{c}}\right], T, \rho\right)$ depends on the value of $T$ or $\rho$ for the prediction. In complete analogy the covariance matrix is obtained for purely predicted properties such as entropy $s$ by Eqs. (39) 


$$
\begin{aligned}
& \operatorname{COV}(p)=J_{p}\left(\left[\theta_{\mathrm{EOS}}^{*}, T_{\mathrm{c}}, \rho_{\mathrm{c}}\right], T, \rho\right) \cdot \operatorname{COV}\left(\left[\theta_{\mathrm{EOS}}^{*}, T_{\mathrm{c}}, \rho_{\mathrm{c}}\right]\right) \cdot J_{p}\left(\left[\theta_{\mathrm{EOS}}^{*}, T_{\mathrm{c}}, \rho_{\mathrm{c}}\right], T, \rho\right)^{\mathrm{T}} \cdot p^{2} \\
& =j_{p}\left(\left[\theta_{\mathrm{EOS}}^{*}, T_{\mathrm{c}}, \rho_{\mathrm{c}}\right], T, \rho\right) \cdot \operatorname{COV}\left(\left[\theta_{\mathrm{EOS}}^{*}, T_{\mathrm{c}}, \rho_{\mathrm{c}}\right]\right) \cdot j_{p}\left(\left[\theta_{\mathrm{EOS}}^{*}, T_{\mathrm{c}}, \rho_{\mathrm{c}}\right], T, \rho\right)^{\mathrm{T}}
\end{aligned}
$$

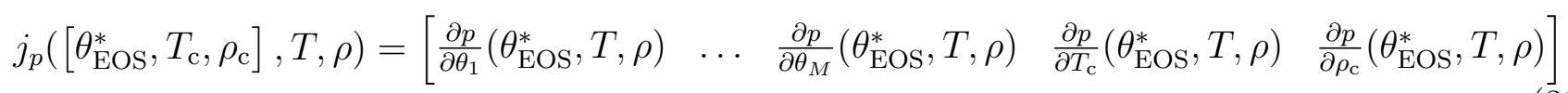

and (40). The covariance matrix of other predicted properties (e.g. $u, h, a, g, \phi$ and $C$ ) can be calculated in a similar manner.

The calculation of the two covariance matrices is numerically not trivial. The parameter covariance matrix $\operatorname{COV}\left(\left[\theta_{\mathrm{EOS}}^{*}, T_{\mathrm{c}}, \rho_{\mathrm{c}}\right]\right)$ is the- 798 oretically obtained through an inversion (see 799 Eq. (33)). However, both the Jacobians and the 800 parameter covariance matrices are very large 801 sparse matrices and the parameter covariance 802 matrix can also be positive semi-definite, which 803 means it has columns that are numerically close 804 to being linearly dependent. We recommend 805 the following procedure to overcome these is- 806 sues:

1. In order to store more significant digits of ${ }^{808}$ matrix elements and to allow more precise ${ }_{810}$ matrix operations compared to ordinary ${ }_{811}$ algebraic calculation of matrices, we rec- ${ }_{812}$ ommend the usage of arbitrary precision ${ }_{813}$ methods, such as mpmath in the Python ${ }_{814}$ programming language.

2. For the calculation of the covariance ma- ${ }^{816}$ trices of the properties, e.g. $C O V(p)$ or ${ }^{817}$ $C O V(s)$, we have used LU decomposi- ${ }^{818}$ tion. The calculation is shown in the sup- ${ }^{819}$ porting information.

Having obtained the respective covariance 822 matrices of the properties, it is now possible 823 to calculate the $95 \%$ confidence interval of the respective properties. Eqs. (41) and (42) allow for a calculation of the $95 \%$ confidence interval for pressure $p$ and entropy $s$. The same holds for the other properties.

$p_{1-\gamma_{t} / 2}^{\text {pred }}=p^{\text {pred }} \pm \sqrt{\operatorname{diag}(C O V(p))} \cdot t\left(n-m, \gamma_{\mathrm{t}} / 2\right)$ $s_{1-\gamma_{t} / 2}^{\text {pred }}=s^{\text {pred }} \pm \sqrt{\operatorname{diag}(C O V(s))} \cdot t\left(n-m, \gamma_{\mathrm{t}} / 2\right)$

It is important to notice that in this study the sum of squared errors and the Jacobian of the fitted properties used relative values, i.e. the residuals and the derivatives have been divided by the corresponding experimental value. This corresponded to the objective function for the fitting of the parameters used by Lemmon et al. ${ }^{12}$ However, this means that the covariance matrices provide the relative uncertainties, which subsequently need to be multiplied with the corresponding predicted thermodynamic property from the EOS, in order to obtain the absolute uncertainty range as shown in Eqs. (41) and (42).

Similar methods can be used to calculate the uncertainties of the differences of properties because covariance of differences or sums of properties can be calculated in a similar manner. This is especially important for properties like $u, s, h, a$ and $g$, in which only the difference of values matters in EOS applications. For example, to calculate the uncertainty of the difference of two entropy values $s_{1}\left(T_{1}, \rho_{1}\right)$ and $s_{2}\left(T_{2}, \rho_{2}\right)$, one can first calculate the Jacobian of the difference between the two values by Eq. (43) and calculate the covariance using 


$$
\begin{gathered}
C O V(s)=j_{s}\left(\left[\theta_{\mathrm{EOS}}^{*}, T_{\mathrm{c}}, \rho_{\mathrm{c}}\right], T, \rho\right) \cdot \operatorname{COV}\left(\left[\theta_{\mathrm{EOS}}^{*}, T_{\mathrm{c}}, \rho_{\mathrm{c}}\right]\right) \cdot j_{s}\left(\left[\theta_{\mathrm{EOS}}^{*}, T_{\mathrm{c}}, \rho_{\mathrm{c}}\right], T, \rho\right)^{\mathrm{T}} \\
j_{s}\left(\left[\theta_{\mathrm{EOS}}^{*}, T_{\mathrm{c}}, \rho_{\mathrm{c}}\right], T, \rho\right)=\left[\frac{\partial s}{\partial \theta_{1}}\left(\theta_{\mathrm{EOS}}^{*}, T, \rho\right) \quad \ldots \quad \frac{\partial s}{\partial \theta_{M}}\left(\theta_{\mathrm{EOS}}^{*}, T, \rho\right) \quad \frac{\partial s}{\partial T_{\mathrm{c}}}\left(\theta_{\mathrm{EOS}}^{*}, T, \rho\right) \quad \frac{\partial s}{\partial \rho_{c}}\left(\theta_{\mathrm{EOS}}^{*}, T, \rho\right)\right]
\end{gathered}
$$

824
Eq. (44).

$$
\begin{aligned}
& j_{s_{1}-s_{2}}\left(\left[\theta_{\mathrm{EOS}}^{*}, T_{\mathrm{c}}, \rho_{\mathrm{c}}\right], T, \rho\right)^{\mathrm{T}} \\
= & {\left[\begin{array}{c}
\frac{\partial s_{1}}{\partial \theta_{1}}\left(\theta_{\mathrm{EOS}}^{*}, T_{1}, \rho_{1}\right)-\frac{\partial s_{2}}{\partial \theta_{1}}\left(\theta_{\mathrm{EOS}}^{*}, T_{2}, \rho_{2}\right) \\
\vdots \\
\frac{\partial s_{1}}{\partial \theta_{m}}\left(\theta_{\mathrm{EOS}}^{*}, T_{1}, \rho_{1}\right)-\frac{\partial s_{2}}{\partial \theta_{m}}\left(\theta_{\mathrm{EOS}}^{*}, T_{2}, \rho_{2}\right) \\
\frac{\partial s_{1}}{\partial T_{\mathrm{c}}}\left(\theta_{\mathrm{EOS}}^{*}, T_{1}, \rho_{1}\right)-\frac{\partial s_{2}}{\partial T_{\mathrm{c}}}\left(\theta_{\mathrm{EOS}}^{*}, T_{2}, \rho_{2}\right) \\
\frac{\partial s_{1}}{\partial \rho_{\mathrm{c}}}\left(\theta_{\mathrm{EOS}}^{*}, T_{1}, \rho_{1}\right)-\frac{\partial s_{2}}{\partial \rho_{\mathrm{c}}}\left(\theta_{\mathrm{EOS}}^{*}, T_{2}, \rho_{2}\right)
\end{array}\right] }
\end{aligned}
$$

The uncertainty of the difference between $s_{1}{ }^{829}$ and $s_{2}$ can be calculated by Eq. (45).

\subsection{Properties with inputs other ${ }_{833}^{832}$ than $T$ and $\rho$}

In practical applications of EOS, there are ${ }^{835}$ many scenarios where the inputs to the EOS ${ }^{836}$ are not temperature and density. For exam- ${ }^{837}$ ple, a user may be given the measured temper- ${ }^{838}$ ature and pressure of a fluid to find the speed of 839 sound, and the user must use an iterative solver ${ }^{840}$ to find the density of the fluid first before calcu- ${ }^{841}$ lating the speed of sound. For substances that ${ }^{842}$ are either superheated vapor or subcooled liq- ${ }^{843}$ uid, if the output variable required is neither ${ }^{844}$ temperature nor density, the calculation of the ${ }^{845}$ uncertainty of the output variable will follow ${ }^{846}$ that in Section 3.1 after calculating the missing ${ }^{847}$ temperature and density values by using an it- ${ }^{848}$ erative solver on the EOS. However, if the out- ${ }^{849}$ put variable is either temperature or density, ${ }^{850}$ the uncertainty of the output variable should ${ }^{851}$ be calculated by propagating the uncertainty of the input variable due to the EOS to the output ${ }_{852}$ using the linearization of the EOS. ${ }^{24 \mid 34}$ This is ${ }_{853}$ done by Eqs. 46 and (47).

$$
\begin{aligned}
& \Delta T^{\text {pred }} \\
= & \left|\left(\left.\frac{\partial T^{\text {pred }}}{\partial x_{1}}\right|_{x_{2}} \Delta x_{1}\right)+\left(\left.\frac{\partial T^{\text {pred }}}{\partial x_{2}}\right|_{x_{1}} \Delta x_{2}\right)\right| \\
= & \left|\left(\left.\frac{\partial \rho^{\text {pred }}}{\partial x_{1}}\right|_{x_{2}} \Delta x_{1}\right)+\left(\left.\frac{\partial \rho^{\text {pred }}}{\partial x_{2}}\right|_{x_{1}} \Delta x_{2}\right)\right|
\end{aligned}
$$

where $\Delta x$ is the standard uncertainty of variable $x, T^{\text {pred }}$ and $\rho^{\text {pred }}$ are the temperature and density values predicted by using an iterative solver with the EOS, $x_{1}$ and $x_{2}$ are the independent variables used to predict $T^{\text {pred }}$ or $\rho^{\text {pred }}$, $\Delta x_{1}$ and $\Delta x_{2}$ are the EOS uncertainties of the variables $x_{1}$ and $x_{2}$ calculated by using the temperature and density values in Eq. (42), and the partial derivatives are obtained according to Thorade and Saadat. 35

If any of $x_{1}$ and $x_{2}$ in Eqs. (46) and (47) are $T$ or $\rho$, their corresponding uncertainties in these equations will be zero.

Since the use of Eqs. 46) and (47) is a result of the difficulty to define covariance of temperature and density, calculating the uncertainties of the sums and differences of temperature and density with equations similar to Eq. (45) is impossible. Hence the calculation of the uncertainties of the sums and differences of temperature and density is the same as that of ordinary variables and follows the method in Kline and McClintock. 24

\subsection{Saturated liquid and vapor properties}

Calculation of thermodynamic properties of a fluid that is based on temperature and density 


$$
\begin{gathered}
C O V\left(s_{1}-s_{2}\right)=j_{s_{1}-s_{2}}\left(\left[\theta_{\mathrm{EOS}}^{*}, T_{\mathrm{c}}, \rho_{\mathrm{c}}\right], T, \rho\right) \cdot \operatorname{COV}\left(\left[\theta_{\mathrm{EOS}}^{*}, T_{\mathrm{c}}, \rho_{\mathrm{c}}\right]\right) \cdot j_{s_{1}-s_{2}}\left(\left[\theta_{\mathrm{EOS}}^{*}, T_{\mathrm{c}}, \rho_{\mathrm{c}}\right], T, \rho\right)^{\mathrm{T}} \\
s_{1,1-\gamma_{\mathrm{t}} / 2}^{\mathrm{pred}}-s_{2,1-\gamma_{t} / 2}^{\mathrm{pred}}=s_{1}-s_{2} \pm \sqrt{\operatorname{diag}\left(C O V\left(s_{1}-s_{2}\right)\right)} \cdot t\left(n-m, \gamma_{\mathrm{t}} / 2\right)
\end{gathered}
$$

values for a homogeneous phase can be achieved 888 by the procedure in Section 3.1. However, users of EOS are often asked for fluid properties of saturated liquid and vapor of a fluid given a temperature or pressure value only. According to Lemmon et al., $\frac{12}{12}$ this is achieved by finding two density values for which Gibbs energy and pressure values are equivalent at the given temperature value - this is the Maxwell's criteria. The smaller density value corresponds to the density of saturated vapor whereas the larger density value corresponds to the density of saturated liquid, and the other properties of ${ }_{889}$ saturated liquid and vapor can be calculated 890 from the density values. The uncertainty of the ${ }_{891}$ thermodynamic properties can be calculated in ${ }_{892}$ the same manner as that in Section 3.1 once ${ }_{893}$ the density values are found. The calculation ${ }_{894}$ of the residuals in the Maxwell criteria is given 895 by Eqs. (48) and 49.

$$
\begin{aligned}
& \Delta r e s_{1}=g\left(\rho_{\mathrm{v}}, T\right)-g\left(\rho_{\mathrm{l}}, T\right) \\
& \Delta r e s_{2}=p\left(\rho_{\mathrm{v}}, T\right)-p\left(\rho_{\mathrm{l}}, T\right),
\end{aligned}
$$

where $\Delta r e s_{1}$ and $\Delta r e s_{2}$ are residual functions of the Maxwell criteria. By finding the density values that lead to zero values for both residual 901 functions, the saturation densities of the fluid ${ }_{902}$ at a certain temperature $T$ can be found. 903

The uncertainty of the Gibbs energy differ- ${ }_{904}$ ence in Eq. (48) given by $\Delta r_{e} s_{1}$ can be calcu- ${ }_{905}$ lated by calculating the covariance of the Gibbs ${ }_{906}$ energy values from Eq. (52).

The uncertainties of the density values can ${ }_{908}$ then be calculated by propagating the uncer- ${ }_{909}$ tainties of the residual functions as shown in ${ }_{910}$
Eqs. (50) and (51).

$$
\begin{aligned}
& \Delta \rho_{\mathrm{l}} \\
= & \left|\left(\left.\frac{\partial r e s_{1}}{\partial \rho_{1}}\right|_{T}\right)^{-1} \Delta r e s_{1}+\left(\left.\frac{\partial r e s_{2}}{\partial \rho_{1}}\right|_{T}\right)^{-1} \Delta r e s_{2}\right| \\
= & \left|\left(\left.\frac{\partial r e s_{1}}{\partial \rho_{\mathrm{v}}}\right|_{T}\right)^{-1} \Delta r e s_{1}+\left(\left.\frac{\partial r e s_{2}}{\partial \rho_{\mathrm{v}}}\right|_{T}\right)^{-1} \Delta r e s_{2}\right|
\end{aligned}
$$

However, $\Delta r e s_{2}$ in Eqs. (50) and (51) involves uncertainty of pressure of saturated liquid that changes very nonlinearly with respect to density. The rapid changes of the sensitivity of saturated liquid pressure with density renders linear error propagation to be invalid to calculate the uncertainties in Eqs. (50) and (51). To calculate $\Delta r e s_{2}$ for the linear error propagation in Eqs. (50) and (51), the uncertainty of the Gibbs energy difference in Eq. (52) is used to approximate the uncertainty of difference of pressure values as shown in Eq. (53).

$$
\Delta r e s_{2}=\frac{p \Delta r e s_{1}}{R T}
$$

Equation (53) is used because the relative difference of Gibbs energy between saturated liquid and vapor changes proportionally with the difference of estimated and measured saturated pressure as shown in Fig. 1.

Since residual functions calculate the differences of Gibbs energy and pressure respectively, the uncertainties of the corresponding Gibbs energy and pressure at saturation are given by the uncertainties of the residual functions as shown in Eqs. (54) and (55). 


$$
\Delta r e s_{1}=\sqrt{\operatorname{diag}\left(C O V\left(g\left(\rho_{\mathrm{v}}, T\right)-g\left(\rho_{\mathrm{l}}, T\right)\right)\right)} \cdot t\left(n-m, \gamma_{\mathrm{t}} / 2\right)
$$

where sat means saturation.

With the approximation in Eq. (53), the un- ${ }_{945}$ certainties of $p$ and $g$ at saturation in Eqs. (54) 946 and (55) and the definition of fugacity, the un- ${ }_{947}$ certainty of fugacity coefficient $\phi$ at saturation 948 can be derived to be zero and the relative un- ${ }_{949}$ certainty of fugacity equals to the relative un- ${ }_{950}$ certainty of pressure at saturation in Eq. (55). ${ }_{951}$

However, other thermodynamic properties at ${ }_{952}$ the saturated states are calculated by solving 953 the original EOS with the density values from ${ }_{954}$ the Maxwell criteria and the original tempera- 955 ture input to the Maxwell criteria. The calcu- ${ }_{956}$ lation would propagate the uncertainties calcu- ${ }_{957}$ lated from Eqs. (50) and (51), and the uncer- ${ }_{958}$ tainties from Eqs. (50) and (51) will be added ${ }_{959}$ to the uncertainties from Eq. (42) to calculate 960 the uncertainties of these thermodynamic prop- ${ }_{961}$ erties. For example, the uncertainty of entropy ${ }_{962}$ of saturated liquid and saturated vapor are cal- ${ }_{963}$ culated by adding the uncertainties as Eqs. (56) 964 and (57).

$$
\Delta s_{\mathrm{l}}=\sqrt{\begin{array}{l}
\operatorname{diag}\left(\operatorname{COV}\left(s\left(T, \rho_{\mathrm{l}}\right)\right)\right) \cdot t\left(n-m, \gamma_{\mathrm{t}} / 2\right)^{2} \\
+\left(\frac{\left.\frac{\partial s_{l}}{\partial T}\right|_{\mathrm{sat}}}{\left.\frac{\partial \rho_{l}}{\partial T}\right|_{\mathrm{sat}}} \Delta \rho_{\mathrm{l}}\right)^{2}
\end{array}}
$$

$$
\Delta s_{\mathrm{v}}=\sqrt{\begin{array}{l}
\operatorname{diag}\left(C O V\left(s\left(T, \rho_{\mathrm{v}}\right)\right)\right) \cdot t\left(n-m, \gamma_{\mathrm{t}} / 2\right)^{2^{669}} \\
+\left(\frac{\left.\frac{\partial s_{\mathrm{v}}}{\partial T}\right|_{\mathrm{sat}}}{\left.\frac{\partial \rho_{\mathrm{v}}}{\partial T}\right|_{\mathrm{sat}}} \Delta \rho_{\mathrm{v}}\right)^{2}
\end{array}}
$$

where 1 means saturated liquid and v is satu- ${ }_{971}$ rated vapor.

When the uncertainty of the differences between the entropy of saturated liquid or vapor and an entropy value in a homogeneous phase

968 is needed, the calculation can be carried out by Eqs. (58) and (59).

The uncertainty of the entropy of vaporization can be calculated by Eq. (60).

Uncertainties of other properties (e.g., $u, h$, $a, c_{p}, c_{v}$ and $\left.\phi\right)$ of saturated liquid and vapor can be calculated in a similar manner as Eqs. (56) and (57). Numerically, the uncertainties can also be calculated from Eq. (42) with density and temperature of the saturated liquid and vapor, but Eq. (42) does not include the uncertainty in the EOS caused by the Maxwell criteria and underestimates the uncertainties. Hence when the uncertainties of properties at saturation are needed, Eqs. (56) and (57) should be used to calculate the uncertainties of entropy of the saturated liquid and vapor instead.

Properties other than temperature can be used as inputs to the EOS to calculate the thermodynamic properties of saturated states; for instance pressure can be used as an input to find the saturation temperature of a fluid at that pressure. To calculate the uncertainty of saturation temperature, the uncertainty of pressure in Eq. (55) can be used to propagate the uncertainty of the EOS to the saturation temperature value, and the uncertainty of saturation temperature can be calculated by Eq. 61.

$$
\Delta T_{\mathrm{sat}}=\left|\left(\left.\frac{\mathrm{d} T}{\mathrm{~d} p}\right|_{\mathrm{sat}}\right) \Delta p_{\mathrm{sat}}\right|
$$
relation as shown in Eq. 62 . 30

$$
\left.\frac{\mathrm{d} T}{\mathrm{~d} p}\right|_{\mathrm{sat}}=\frac{1 / \rho_{\mathrm{l}}-1 / \rho_{\mathrm{v}}}{s_{\mathrm{v}}-s_{\mathrm{l}}}
$$

and the uncertainty in vapor pressure $\Delta p_{\text {sat }}$ is obtained from Eq. (53). 


$$
\begin{aligned}
& \Delta\left(s_{\mathrm{l}}-s_{1 \mathrm{p}}\right)=\sqrt{\operatorname{diag}\left(C O V\left(s\left(T, \rho_{\mathrm{l}}\right)-s_{1 \mathrm{p}}\left(T_{1 \mathrm{p}}, \rho_{1 \mathrm{p}}\right)\right)\right) \cdot t\left(n-m, \gamma_{\mathrm{t}} / 2\right)^{2}+\left(\frac{\left.\frac{\partial s_{1}}{\partial T}\right|_{\mathrm{sat}}}{\left.\frac{\partial \rho_{1}}{\partial T}\right|_{\text {sat }}} \Delta \rho_{\mathrm{l}}\right)^{2}} \\
& \Delta\left(s_{\mathrm{v}}-s_{1 \mathrm{p}}\right)=\sqrt{\operatorname{diag}\left(\operatorname{COV}\left(s\left(T, \rho_{\mathrm{v}}\right)-s_{1 \mathrm{p}}\left(T_{1 \mathrm{p}}, \rho_{1 \mathrm{p}}\right)\right)\right) \cdot t\left(n-m, \gamma_{\mathrm{t}} / 2\right)^{2}+\left(\frac{\left.\frac{\partial s_{\mathrm{v}}}{\partial T}\right|_{\mathrm{sat}}}{\left.\frac{\partial \rho_{\mathrm{v}}}{\partial T}\right|_{\mathrm{sat}}} \rho_{\mathrm{v}}\right)^{2}}
\end{aligned}
$$

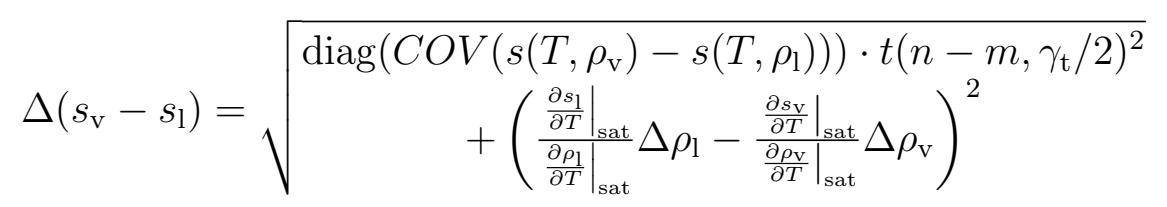

\subsection{Thermodynamic quality}

When defining the thermodynamic state of liquid and vapor phases in equilibrium of a pure substance, an additional property called thermodynamic quality as defined by Eq. 63).

$$
q=\frac{1 / \rho-1 / \rho_{\mathrm{l}}(T)}{1 / \rho_{\mathrm{v}}(T)-1 / \rho_{\mathrm{l}}(T)}
$$

where $q$ is thermodynamic quality, $\rho_{\mathrm{l}}$ is the 994 density of saturated liquid, $\rho_{\mathrm{v}}$ is the density of 995 saturated vapor, $q$ is the thermodynamic qual- 996 ity, and $\rho_{\mathrm{v}} \leq \rho \leq \rho_{\mathrm{l}}$.

Although $q$ is not given as part of an out- 998 put of a part of Helmholtz-based EOS, it is 999 commonly computed as an internal function in $_{1000}$ software packages calculating thermodynamic ${ }_{1001}$ properties, and hence it is necessary to define its ${ }_{1002}$ uncertainty calculation. From Eq. 63), it can be seen that the uncertainty of thermodynamic quality due to EOS mainly comes from the cal- ${ }^{1003}$ culation of the density of the saturated liquid ${ }^{1004}$ and vapor. By propagating the uncertainty of ${ }_{1005}$ the density of saturated liquid and vapor, the ${ }_{1006}$ uncertainty of thermodynamic quality due $\mathrm{to}_{1007}$ the EOS can be calculated by Eq. (64).

$$
\Delta q=\left|\frac{\partial q}{\partial \rho_{\mathrm{v}}} \Delta \rho_{\mathrm{v}}+\frac{\partial q}{\partial \rho_{\mathrm{l}}} \Delta \rho_{\mathrm{l}}\right|
$$

$$
\begin{aligned}
\frac{\partial q}{\partial \rho_{\mathrm{l}}} & =\frac{1-q}{\rho_{\mathrm{l}}^{2}\left(\frac{1}{\rho_{\mathrm{v}}}-\frac{1}{\rho_{\mathrm{l}}}\right)} \\
\frac{\partial q}{\partial \rho_{\mathrm{v}}} & =\frac{q}{\rho_{\mathrm{v}}^{2}\left(\frac{1}{\rho_{\mathrm{v}}}-\frac{1}{\rho_{\mathrm{l}}}\right)}
\end{aligned}
$$

where $\Delta q$ is the uncertainty of the thermodynamic quality, and $\Delta \rho_{\mathrm{v}}$ and $\Delta \rho_{\mathrm{l}}$ are uncertainties calculated in Section 3.3 .

If either temperature or density are not given as inputs to the EOS, they will be first calculated by solving the EOS iteratively. The temperature and density values will be used to calculate uncertainty of the thermodynamic quality according to Eq. (64).

\subsection{Other thermodynamic prop- erties of two-phase mixtures}

The calculation method of the uncertainty of the property from the EOS for two-phase systems with input variables other than temperature and density is different from that in Sections 3.1 and 3.2 , because it involves the calculation of the thermodynamic quality. In this case, the uncertainties calculated in Section 3.3 related to the Maxwell criteria are also involved in the calculation of the uncertainties of proper- 
ties using thermodynamic quality as one of the 1044 inputs.

When the thermodynamic quality is 0 (saturated liquid) or 1 (saturated vapor), the uncertainty of the properties can be calculated based on the equations in Section 3.3, and their uncertainties are given by Eqs. (54), (55) and (61).1045 However, for all other properties, their uncer-1046 tainties are calculated with a different method.1047 For example, if the uncertainty of an entropy1048 value is calculated with an intermediate thermodynamic property as one of its inputs, to in- ${ }_{1049}$ clude the uncertainty propagated from the use of the Maxwell criteria, its calculation will be ${ }^{1050}$ carried out with Eq. (67).

$$
\begin{aligned}
& =\sqrt[\begin{array}{l}
\left(\left.\frac{\partial s_{2 \mathrm{p}}}{\partial q}\right|_{T} \Delta q\right)^{2} \\
+\operatorname{diag}\left(C O V\left(s_{2 \mathrm{p}}\right)\right) \cdot t\left(n-m, \gamma_{\mathrm{t}} / 2\right)^{2}
\end{array}]{=\sqrt{\begin{array}{l}
\left.\left[s_{\mathrm{v}}-s_{\mathrm{l}}\right) \Delta q\right]^{2} \\
+\operatorname{diag}\left(C O V\left(s_{2 \mathrm{p}}\right)\right) \cdot t\left(n-m, \gamma_{\mathrm{t}} / 2\right)^{2}
\end{array}}} \\
& =
\end{aligned}
$$

where the subscript 2p means two-phase fluid. ${ }_{1062}$

For the uncertainty of its difference with en- ${ }_{1063}$ tropy values in single phase, the uncertainty can ${ }_{1064}$ be calculated by Eq. (68).

$$
=\sqrt{\begin{array}{l}
{\left[\left(s_{2 \mathrm{p}}\left(T_{2 \mathrm{p}}, q_{2 \mathrm{p}}\right)-s_{1 \mathrm{p}}\right)\right.} \\
\left.\left.+\operatorname{diag}\left(C O V\left(T_{2 \mathrm{p}}\right)-s_{\mathrm{l}}\left(T_{2 \mathrm{p}}\right)\right) \Delta s_{2 \mathrm{p}}-s_{1 \mathrm{p}}\right)\right) \\
\cdot t\left(n-m, \gamma_{\mathrm{t}} / 2\right)^{2}
\end{array}}
$$

1070

where $1 \mathrm{p}$ is single phase.

When the uncertainty of its difference with ${ }_{1074}^{1073}$ entropy values of the saturated liquid or vapor ${ }_{1075}^{1074}$ is needed, the uncertainty can be calculated by ${ }_{1076}$ Eqs. (69) and (70).

The uncertainty of the difference between $\mathrm{a}_{1078}^{1077}$ pair of two-phase entropy states can be calcu- ${ }^{1079}$ lated by Eq. (71). The uncertainty of properties $u, h$ and $a$ can also be calculated in a manner ${ }_{1081}^{1080}$ similar to Eqs. (67) to (70).

The uncertainty of density can be calculated ${ }_{1083}^{1082}$ from

$$
\Delta \rho_{2 \mathrm{p}}=\sqrt{\left(\frac{-1 / \rho^{2}}{1 / \rho_{\mathrm{v}}-1 / \rho_{\mathrm{l}}} \Delta q\right)^{2}+(\Delta \rho)^{2}},
$$

where $\Delta \rho$ comes from Eq. (47).

The properties $c_{p}$ and $w$ are undefined for two-phase states and therefore do not have any uncertainty values associated with them.

\subsection{Summary}

Figure 2 summarizes the choice of uncertainty calculation method of the EOS based on the input variables and the phase of the fluid where the uncertainty of a property value is needed.

When the input variables are temperature and density, the calculation method follows the basic uncertainty calculation method derived in Section 3.1 because the calculation does not involve the determination of the phase of the fluid nor any iterative calculation. If the input variables are not temperature and density, the EOS would involve iterative calculation. For superheated vapor and subcooled liquid, the method should follow Section 3.2. For saturated vapor and liquid, the calculation involves the Maxwell criteria and Section 3.3 should be followed. For two-phase states, if the output is Gibbs energy, pressure, fugacity coefficient or temperature, the calculation method would be the same as that of saturated liquid and vapor, and the method in Section 3.3 should be followed. If the output is thermodynamic quality, the uncertainty calculation method in Section 3.4 should be used. Otherwise, the uncertainty calculation method in Section 3.5 should be followed.

When the differences of properties are needed, the calculation steps of the uncertainties follow the flowchart in Fig. 3

If the difference involves $T, \rho$, or $q$, whose covariance cannot be calculated directly, the uncertainty of the differences will be calculated by propagating the uncertainties of individual property values with methods in Kline and McClintock. ${ }^{24}$ If the inputs are all $T$ and $\rho$, no Maxwell criteria will be involved even for two- 


$$
\begin{aligned}
& \Delta\left(s_{2 \mathrm{p}}\left(T_{2 \mathrm{p}}, q_{2 \mathrm{p}}\right)-s_{1}\left(T_{1}\right)\right)=\sqrt{\begin{array}{l}
\operatorname{diag}\left(\operatorname{COV}\left(s_{2 \mathrm{p}}\left(T_{2 \mathrm{p}}, q_{2 \mathrm{p}}\right)-s_{\mathrm{l}}\left(T_{1}\right)\right)\right) \cdot t\left(n-m, \gamma_{\mathrm{t}} / 2\right)^{2} \\
+\left(\frac{\left.\frac{\partial s_{1}\left(T_{1}\right)}{\partial T}\right|_{\mathrm{sat}}}{\left.\frac{\partial \rho_{\mathrm{l}}\left(T_{1}\right)}{\partial T}\right|_{\mathrm{sat}}} \rho_{\mathrm{l}}\left(T_{1}\right)-\left[\left(s_{\mathrm{v}}\left(T_{2 \mathrm{p}}\right)-s_{1}\left(T_{2 \mathrm{p}}\right)\right) \Delta q_{2 \mathrm{p}}\right]\right)^{2}
\end{array}} \\
& \Delta\left(s_{2 \mathrm{p}}\left(T_{2 \mathrm{p}}, q_{2 \mathrm{p}}\right)-s_{\mathrm{v}}\left(T_{\mathrm{v}}\right)\right)=\sqrt{\begin{array}{l}
\operatorname{diag}\left(\operatorname{COV}\left(s_{2 \mathrm{p}}\left(T_{2 \mathrm{p}}, q_{2 \mathrm{p}}\right)-s_{\mathrm{v}}\left(T_{\mathrm{v}}\right)\right)\right) \cdot t\left(n-m, \gamma_{\mathrm{t}} / 2\right)^{2} \\
+\left(\frac{\left.\frac{\partial s_{\mathrm{v}}\left(T_{\mathrm{v}}\right)}{\partial T}\right|_{\mathrm{sat}}}{\left.\frac{\partial \rho_{\mathrm{v}}\left(T_{\mathrm{v}}\right)}{\partial T}\right|_{\mathrm{sat}}} \Delta \rho_{\mathrm{v}}\left(T_{\mathrm{v}}\right)-\left[\left(s_{\mathrm{v}}\left(T_{2 \mathrm{p}}\right)-s_{\mathrm{l}}\left(T_{2 \mathrm{p}}\right)\right) \Delta q_{2 \mathrm{p}}\right]\right)^{2}
\end{array}}
\end{aligned}
$$

phase substances, and the uncertainty of prop-1107 erty differences can be calculated directly following Section 3.1. If the difference involves ${ }^{1108}$ two-phase states, the property calculation fol- ${ }^{1109}$ lows that listed in Section 3.5. If the difference ${ }^{1110}$ involves saturated liquid or vapor, the method ${ }^{111}$ to calculate the uncertainty of property differ- ${ }^{1112}$ ences in Section 3.3. Otherwise, the calculation ${ }^{113}$ method of uncertainty methods listed in Sec- ${ }^{1114}$ tion 3.1 will be used.

The Python code used to calculate the uncer- ${ }^{1116}$ tainties is listed in the Supplementary Materials ${ }^{1117}$ for reference.

\section{Results and Discussion}

To illustrate the application of the proposed un-1123 certainty calculation method, it is applied to1124 the EOS of propane. ${ }^{12}$ Its EOS is Helmholtz-1125 energy-explicit, and the proposed uncertainty 1126 calculation method can be applied to it to1127 demonstrate how uncertainties of the EOS af-1128 fect the properties estimated by the EOS. $\quad{ }_{1129}$

\subsection{Experimental data}

The experimental data used in this work were obtained via NIST ThermoDataEngine (TDE) \#103b. $\stackrel{36}{39}$ The data sources are summarized in Table 3, and a more detailed list of the experimental data considered is provided in the Supporting Data. These data span several different types, including densities, saturation properties (vapor pressure, latent heat of vaporization, etc.) and properties in homogeneous phases (speed of sound, heat capacities, etc.).

The resultant normalized $\sigma$ values in Eq. (26) that are calculated based on the deviations between the data in Table 3 and the estimation from the EOS are listed in Table 4. These $\sigma$ values come from the relative deviations between the EOS and the measurement results. The relative deviation between measurement data and the EOS estimation of pressure from the data sets of liquid and vapor density yield the largest values in Table 4. It can be seen that the major source of uncertainties come from the deviations of the measurement data of density of liquid and vapor with the EOS estimation. The relative deviations between measurement and

$$
\Delta\left(s_{2 \mathrm{p}, 1}\left(T_{2 \mathrm{p}, 1}, q_{2 \mathrm{p}, 1}\right)-s_{2 \mathrm{p}, 2}\left(T_{2 \mathrm{p}, 2}, q_{2 \mathrm{p}, 2}\right)\right)=\sqrt{\begin{array}{l}
\operatorname{diag}\left(C O V\left(s_{2 \mathrm{p}, 1}-s_{2 \mathrm{p}, 2}\right)\right) \cdot t\left(n-m, \gamma_{\mathrm{t}} / 2\right)^{2} \\
+\left(\left[\left(s_{\mathrm{v}}\left(T_{2 \mathrm{p}, 1}\right)-s_{\mathrm{l}}\left(T_{2 \mathrm{p}, 1}\right)\right) \Delta q_{2 \mathrm{p}, 1}\right]-\left[\left(s_{\mathrm{v}}\left(T_{2 \mathrm{p}, 2}\right)-s_{\mathrm{l}}\left(T_{2 \mathrm{p}, 2}\right)\right) \Delta q_{2 \mathrm{p}, 2}\right]\right)^{2}
\end{array}}
$$




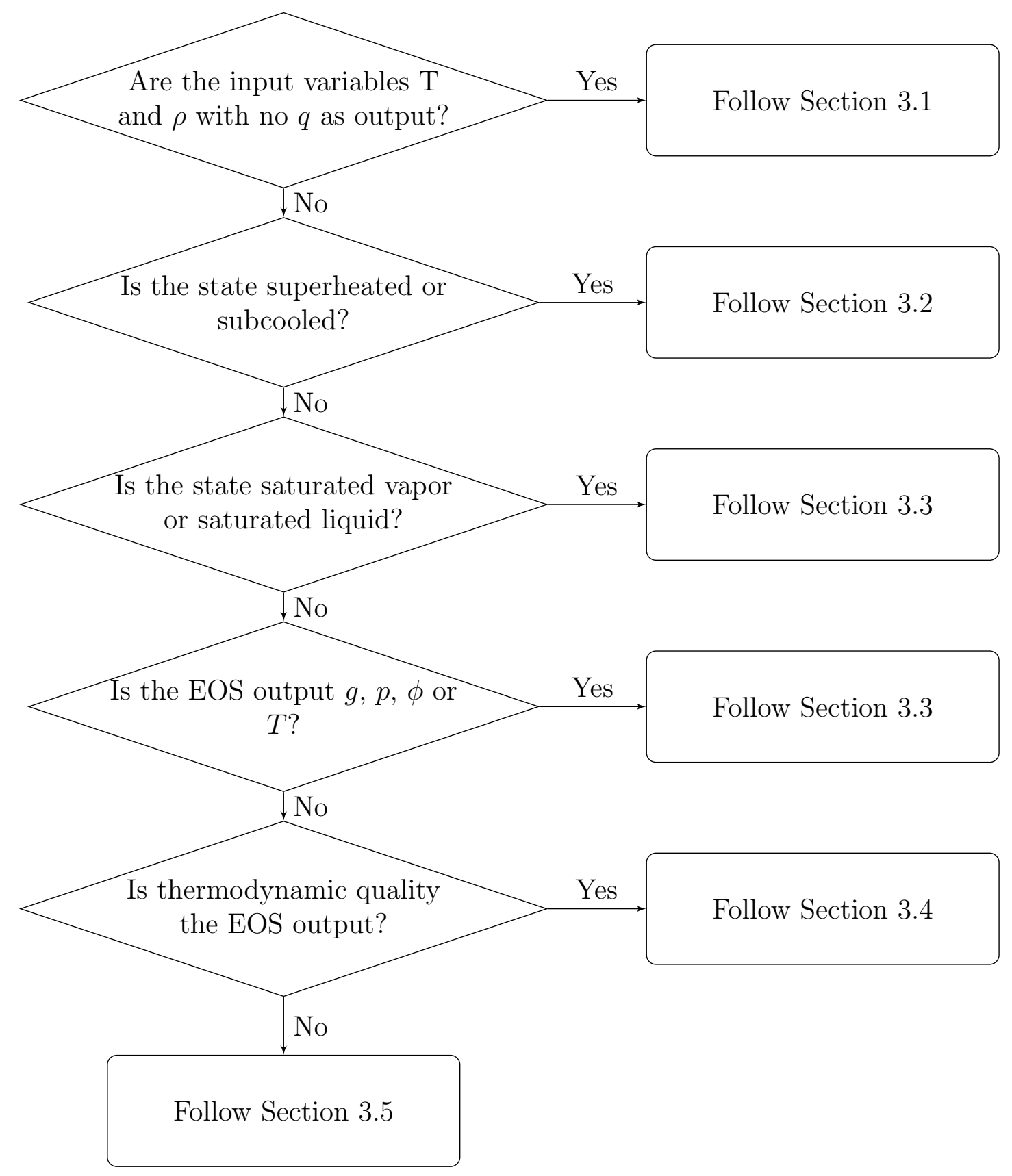

Figure 2: Flowchart showing the choice of equations to calculate the uncertainties of EOS based on the input variables and state of the fluid 


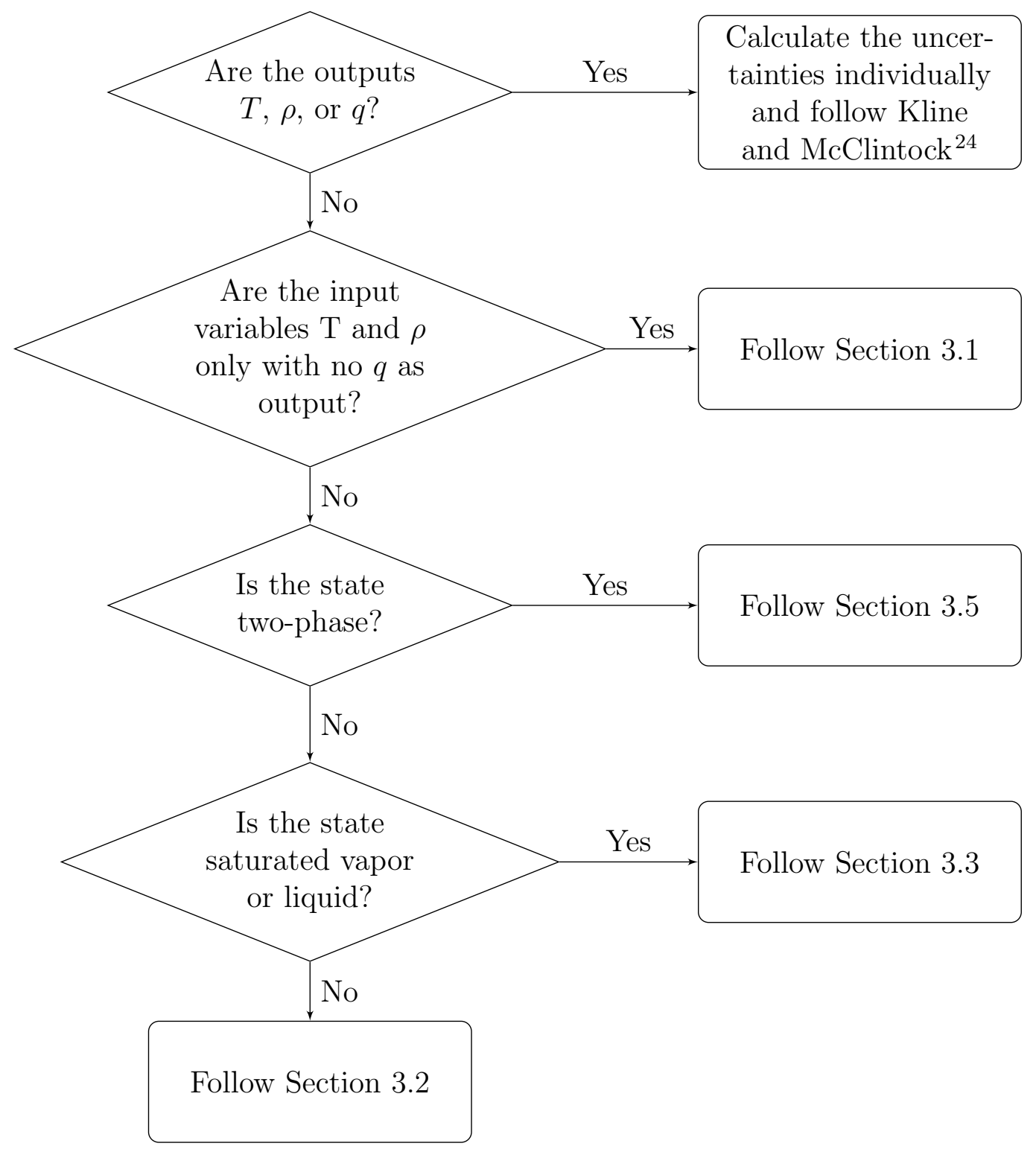

Figure 3: Flowchart showing the choice of equations to calculate the uncertainties of property differences

the EOS estimation of specific heat capacity 1146 of liquid and vapor, saturation pressure, sec-1147 ond virial coefficient and speed of sound also1148 contribute to the uncertainties of the EOS sig-1149 nificantly. However, the relative deviations be-1150 tween measurement and the EOS estimation of ${ }_{1151}$ ideal gas specific heat capacity, critical temper-1152 ature and critical density are much smaller than ${ }_{1153}$ that of the other properties, and they do not ${ }_{1154}$ contribute to the uncertainty of the EOS as 1155 significantly as the measurement of the other ${ }_{1156}$ properties.

Table 4 also shows the corresponding stan-1158 dard measurement uncertainty of different ${ }_{1159}$ property values that are converted. These uncertainty values were taken from ThermoDataEngine and represent a combination of experimental uncertainties ascribed by the individual researcher as well as, when appropriate, expert evaluation to increase the uncertainty to a more reasonable value if the claimed uncertainty is not reasonable. These standard uncertainty values are calculated by averaging the standard measurement uncertainties of the measurement data, and they can be compared directly with the normalized $\sigma$ values. The results show that the noramlized $\sigma$ are not significantly larger than their corresponding 
Table 3: Summary of experimental data from TDE considered in this study. Any experimental data points flagged by TDE as being unreliable were not included.

\begin{tabular}{cccc}
\hline Data type & $N$ & $\begin{array}{c}T \text { range } \\
\mathrm{K}\end{array}$ & $\begin{array}{c}p \text { range } \\
\mathrm{MPa}\end{array}$ \\
\hline$T_{\mathrm{c}}$ & 45 & & \\
$p_{\mathrm{c}}$ & 38 & & \\
$\rho_{\mathrm{c}}$ & 18 & & \\
$p_{\text {sat }}$ & 1392 & 85 to 369 & $1.6332 \times 10^{-10}$ to 4.91426 \\
Liquid $\rho$ & 3746 & 88 to 369 & 0.3546 to 1072.7 \\
Saturated Liquid $\rho$ & 528 & 86 to 369 & \\
Vapor $\rho$ & 3494 & 243 to 609 & 0.040934 to 1047 \\
Saturated Vapor $\rho$ & 100 & 230 to 369 & \\
Second Virial Coefficient $B$ & 167 & 183 to 559 & \\
Enthalpy of Vaporization & 50 & 186 to 362 & \\
Liquid $c_{p}$ & 108 & 89 to 366 & 0.101325 to 5.43 \\
Vapor $c_{p}$ & 138 & 273 to 573 & 0.049033 to 10.3421 \\
$c_{p 0}$ & 50 & 148 to 353 & \\
Liquid $w$ & 110 & 100 to 374 & \\
Homogeneous Phase $c_{p}$ & 655 & 90 to 339 & 1.92 to 60.58 \\
Vaturated Vapor $w$ & 59 & 90 to 325 & \\
Homogenous Phase $w$ & 593 & 239 to 498 & $9.80665 \times 10^{-6}$ to 101.337 \\
\hline
\end{tabular}

measurement uncertainties with the exception ${ }_{1182}$ of the second virial coefficient and the speed of ${ }_{1183}$ sound. For these two variables, the normalized ${ }_{1184}$ $\sigma$ are at least $100 \%$ larger than their measure-1185 ment counterparts. The normalized $\sigma$ for $p_{1186}$ from data sets of liquid and vapor $\rho$ is also1187 much larger than the relative standard mea-1188 surement uncertainty of $p$ values at saturation.1189 The results show that the inaccuracy of $\mathrm{EOS}_{1190}$ estimation may also play a critical role in the ${ }_{1191}$ uncertainty of EOS besides the measurement ${ }_{1192}$ uncertainty of the experimental data.

We present the results in three parts. First ${ }^{1193}$ the parameter identifiability is analyzed. Then ${ }_{1195}^{1194}$ the uncertainties of saturation properties are studied. Finally the variation of uncertainties ${ }^{1196}$ with the thermodynamic state points are pre-1197 sented and discussed.

\subsection{Covariance, correlation ma- ${ }^{1200}$ trix and identifiability}

Considering the parameter covariance matrix ${ }^{1203}$ (as calculated by Eq. 9) it is also possible ${ }^{1204}$ to assess the parameter identifiability. The square-root of the diagonal elements of the covariance matrix (i.e. $\left.\sqrt{\operatorname{diag}\left(\operatorname{COV}\left(\theta_{\mathrm{EOS}}^{*}\right)\right)}\right)$ correspond to the parameter standard deviation. If the standard deviations with respect to the parameter values are large, parameters are not practically identifiable. This means that there are not enough data to estimate the parameters with the current model and objective function. We chose as a metric for practical identifiability: $\left|\sqrt{\operatorname{diag}\left(\operatorname{COV}\left(\theta_{\mathrm{EOS}}^{*}\right)\right)} / \theta_{\mathrm{EOS}}^{*}\right| \cdot \operatorname{In}$ the given study nearly all the parameters (besides $n_{16}$ and $n_{17}$ ) have a small standard deviation compared to the parameter value, i.e. $\left|\sqrt{\operatorname{diag}\left(\operatorname{COV}\left(\theta_{\mathrm{EOS}}^{*}\right)\right)} / \theta_{\mathrm{EOS}}^{*}\right|<0.1$ (see the Supporting Information). This result implies that the amount of experimental data used for the parameter fitting (see Lemmon et al. ${ }^{12}$ ) and the uncertainty analysis is sufficiently high to guarantee the identification of the parameters from the data. A small amount of experimental data would lead to poor identifiability and therefore parameter values with large standard 
Table 4: Normalized $\sigma$ values from deviations between measurement and EOS estimation in Eq. (26) for propane, and their corresponding average relative standard measurement uncertainty

\begin{tabular}{lll}
\hline Property & Normalized $\sigma$ & $\begin{array}{l}\text { Corresponding average rela- } \\
\text { tive standard uncertainty }\end{array}$ \\
\hline$c_{p 0}$ & $5.104 \times 10^{-3}$ & $1.448 \%$ \\
$c_{p}$ & $8.113 \times 10^{-2}$ & $4.391 \%$ \\
$c_{v}$ & $7.021 \times 10^{-2}$ & $1.081 \%$ \\
Saturated liquid & $2.661 \times 10^{-2}$ & $5.228 \%$ \\
and vapor $p$ & & \\
$p$ from data sets of & $1.465 \times 10^{-1}$ & Not available \\
liquid and vapor $\rho$ & & \\
$\rho_{c}$ & $4.447 \times 10^{-6}$ & $1.306 \%$ \\
$T_{c}$ & $7.767 \times 10^{-6}$ & $0.100 \%$ \\
$B$ & $3.880 \times 10^{-2}$ & $1.623 \%$ \\
$w$ & $6.388 \times 10^{-2}$ & $0.196 \%$ \\
\hline
\end{tabular}

deviations. It is recommended that develop-1234 ers of Helmholtz-type EoS analyze the parame-1235 ter covariance matrix with respect to parameter ${ }_{1236}$ identifiability in order to ensure that sufficient ${ }_{1237}$ experimental data has been used for the fitting ${ }_{1238}$ of the respective parameters.

1239

The parameter correlation matrix (obtained ${ }_{1240}$ by Eq. 10), contains information of the corre-1241 lation coefficients between the parameters; the ${ }_{1242}$ parameter correlation matrix is attached in the ${ }_{1243}$ supplementary material. Several of the parame-1244 ters are highly correlated, corresponding to high ${ }_{1245}$ correlation coefficients $> \pm 0.7$ (a correlation cO-1246 $^{-124}$ efficient equal to 1 would correspond to a per-1247 fect correlation). This means that many param-1248 eters are not independent from each other, due ${ }_{1249}$ to the structure of the equations: Parameters1250 increase or decrease, when other ones increase ${ }_{1251}$ or decrease. However, due to the fact that a suf-1252 ficiently large amount of experimental data has1253 been used for the calculation, the high correla-1254 tion coefficients did not lead to high parameter ${ }_{1255}$ uncertainties.

1256

1257

\subsection{Computational cost to calcu- ${ }^{1250}$ late an uncertainty \\ 1259 1260}

To examine if the computational cost of the ${ }^{1261}$ proposed method is lower than the alterna-1262 tive methods, $\frac{122}{122}$ the computational cost of ${ }^{1263}$ the methods are analyzed by comparing the ${ }^{1264}$ most computational expensive portion of the methods. In the alternative methods, 1122 the most computationally expensive step lies in the Monte Carlo method which requires at least 1000 computations of the EOS before the convergence for each uncertainty value. In contrast, the most computational expensive portion of the method is the calculation of the Jacobian vector in Eq. (11). While the Jacobian matrix in Eq. (32) and the Covariance matrix in Eq. (35) are more computationally expensive than the calculation of a Jacobian vector, their values, upon calculation from training data, are stored in memory for all other uncertainty calculation of properties of the same substance. Their calculation does not repeat, and the computational cost is negligible compared to the computational cost of the uncertainty method in the long run. In this study, each entry of the Jacobian vector is calculated by performing differentiation on the EOS, and the computational cost of each entry of the vector is at most three times of the computational cost of a computation of the EOS. The number of entries in each Jacobian vector equals to the number of parameters in an EOS plus two as shown in Eq. (38) (i.e. 99 for propane in this study), and the computational cost of a Jacobian vector is at most 300 computations of the EOS. Thus the computational cost of the proposed method is around $30 \%$ of the computational cost of the alternative 
methods, and the computation of the proposed method is faster than the alternatives.

\subsection{Saturated property uncer- tainty}

To understand the magnitude of uncertainties of various properties within the applicable range of the EOS, the uncertainties of density and enthalpy along the saturation curves are plotted from the triple point temperature of $85.525 \mathrm{~K}$ to the critical temperature ${ }^{12}$ with uncertainty bounds overlaid as shown in Fig. 4

The uncertainties in Figure 4 are reasonable at most conditions except near the critical point in both diagrams and at low temperature for saturated vapor in Fig. 4a. The uncertainties of properties near the critical point are much larger than ones further from the critical point, which is also reported in Lemmon et al. ${ }^{12}$ without quantification. The reason for the large uncertainties is due to the mathematical structure of the Maxwell criteria as shown in Section 3.3 According to the Maxwell criteria and Eqs. (50) and (51), the uncertainties of the density of saturated liquid and vapor and hence the uncertainties of other saturated properties depend on the derivative of densities with respect to pressure and Gibbs energy. As the temperature of a saturated substance approaches the critical point, the saturation densities change rapidly along the saturation line, and the derivatives of densities with respect to pressure become very large in magnitude, as shown in Fig. 5.

The trend of the derivative increasing to infinity at the critical temperature as shown in Fig. 5 are unavoidable because critical points are classically defined as the point where $\left.\frac{\partial \rho}{\partial p}\right|_{T}$ and $\left.\frac{\partial^{2} \rho}{\partial p^{2}}\right|_{T}$ are infinite. $\frac{30}{30}$ The uncertainties of saturated liquid and vapor properties depend on the uncertainties of their densities; hence their large derivatives with respect to pressure near the critical point results in large uncertainties as shown in Fig. 4.

Fig. 5 also explains why the uncertainty of ${ }_{1312}^{1311}$ saturated vapor is large at low temperature in ${ }^{1312}$ Fig. 4a. The relative derivatives of density of ${ }^{1313}$ saturated vapor with respect to pressure is in-

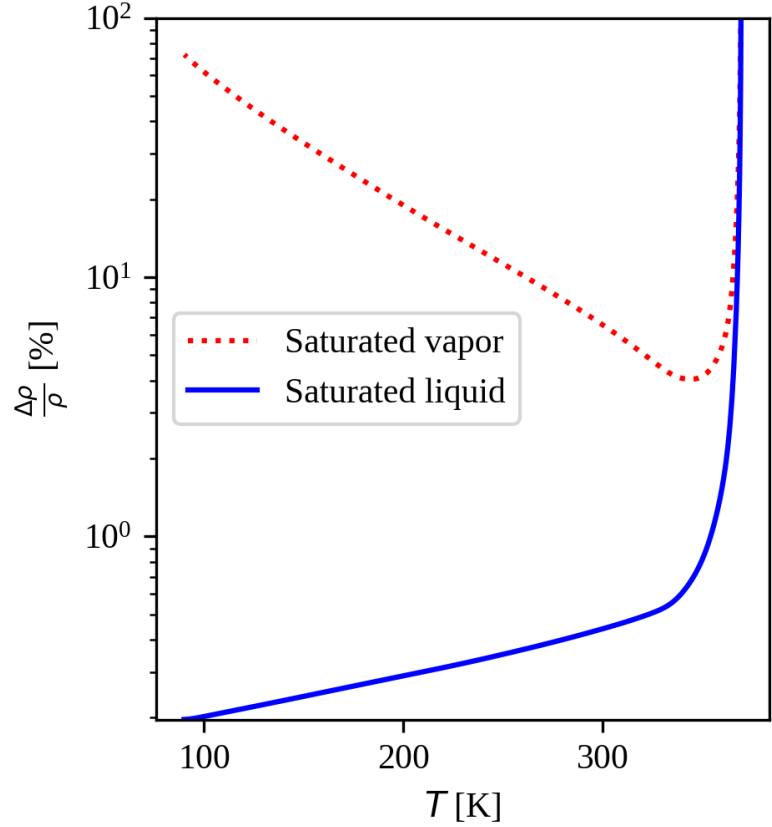

(a) Change of relative uncertainty of $\rho$ of saturated liquid and vapor with temperature along the saturation line

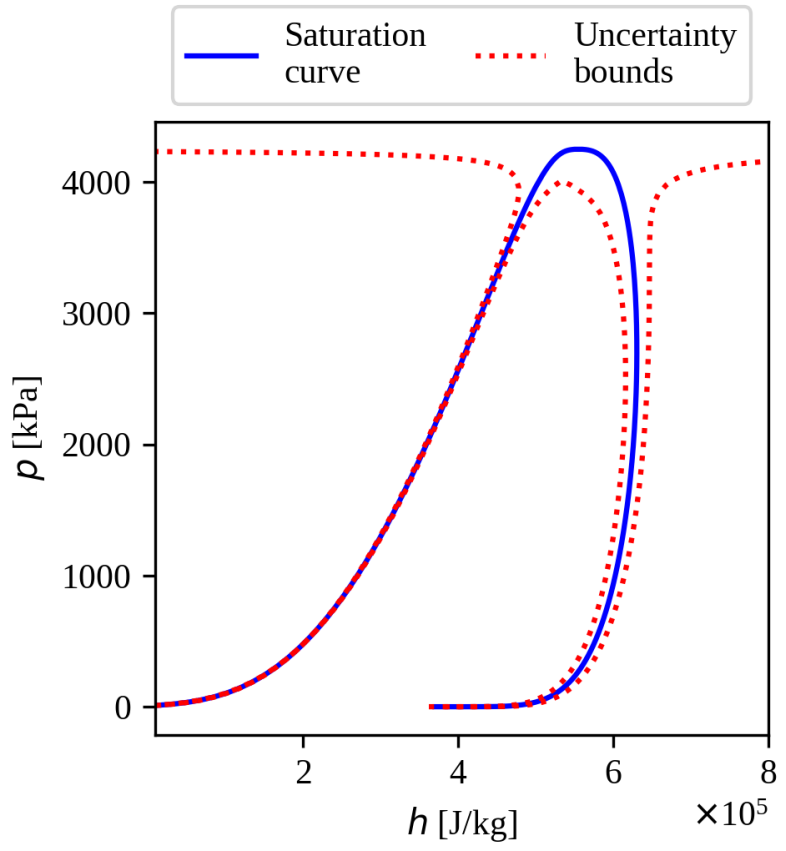

(b) $p$ - $h$ diagram with uncertainties of $h$ relative to its reference state

Figure 4: Uncertainties of properties of propane along saturation lines in different property diagrams from $85.525 \mathrm{~K}$ to its critical temperature

creasing with decreasing temperature. These increasing derivatives propagate into the uncertainty of saturated density by Maxwell criteria, and the uncertainty of density of saturated 


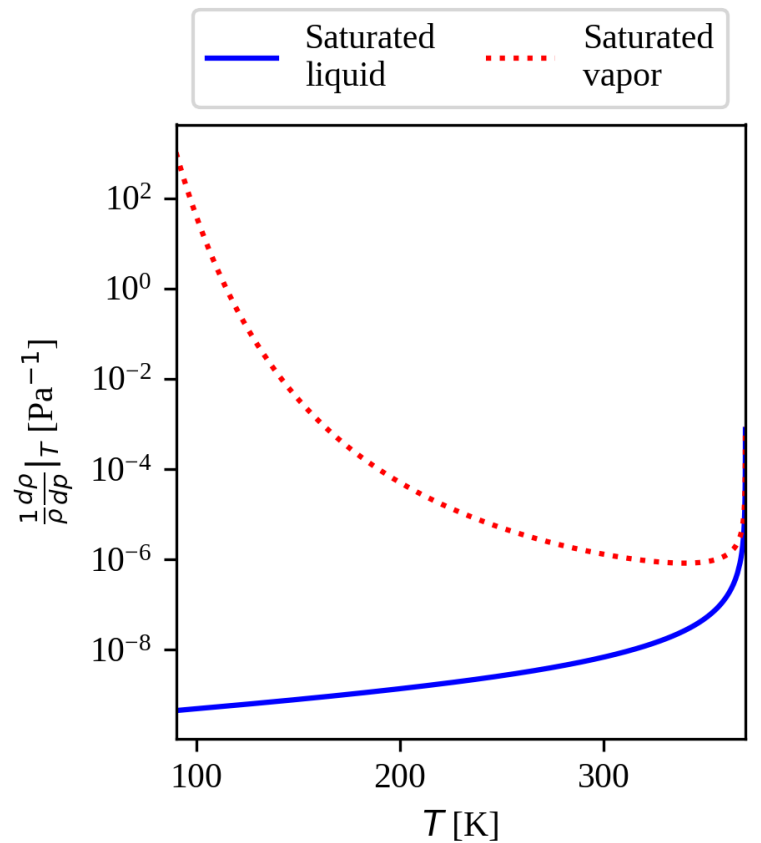

Figure 5: Changes of relative derivatives of density of pressure with respect to pressure with temperature from $85.525 \mathrm{~K}$ to the critical temperature of propane vapor becomes large. In contrast, the relative derivative of density of saturated liquid is decreasing with decreasing temperature, and by the same uncertainty propagation mechanism, the uncertainty of density of saturated liquid becomes small at low temperature in Fig. 4a.

To examine the uncertainty of saturation pressure along the saturation line, the uncertainty of saturation temperature and the relative uncertainty of pressure calculated by Eq. (73) are plotted with the saturation properties in Fig. 6.

Relative uncertainty of variable $x=\frac{\Delta x}{x}$

Figure 6a shows that the uncertainty of saturation temperature becomes more significant at lower temperature. At temperature around $200 \mathrm{~K}$, the uncertainty peaks at around $1.5 \mathrm{~K}$, and it approaches $1 \mathrm{~K}$ as the temperature is ${ }_{1336}$ lowered to the triple point of propane. How- ${ }_{1337}$ ever, when the uncertainty is studied from the $\mathrm{f}_{1338}$ perspective of pressure in Figure 6b, the relative ${ }_{1339}$

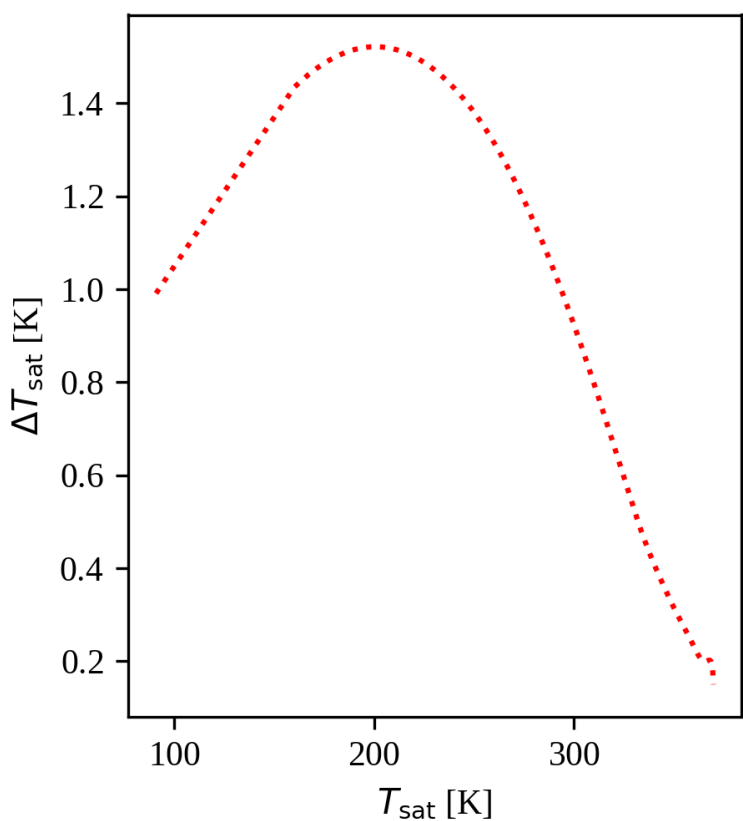

(a) Changes of uncertainty of saturation temperature with temperature

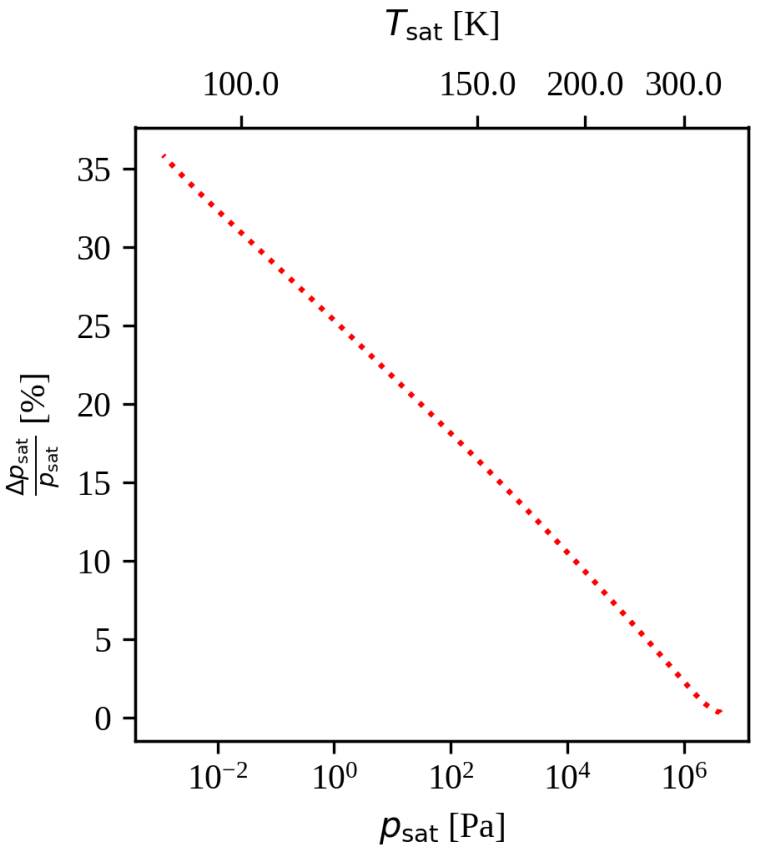

(b) Changes of relative uncertainty of saturation pressure with pressure

Figure 6: Changes of saturation property uncertainties with their corresponding properties of propane

uncertainty of saturation pressure is found to be increasing as the pressure drops. The cause of the large uncertainty is due to the scattering of the deviations between the estimated and mea- 


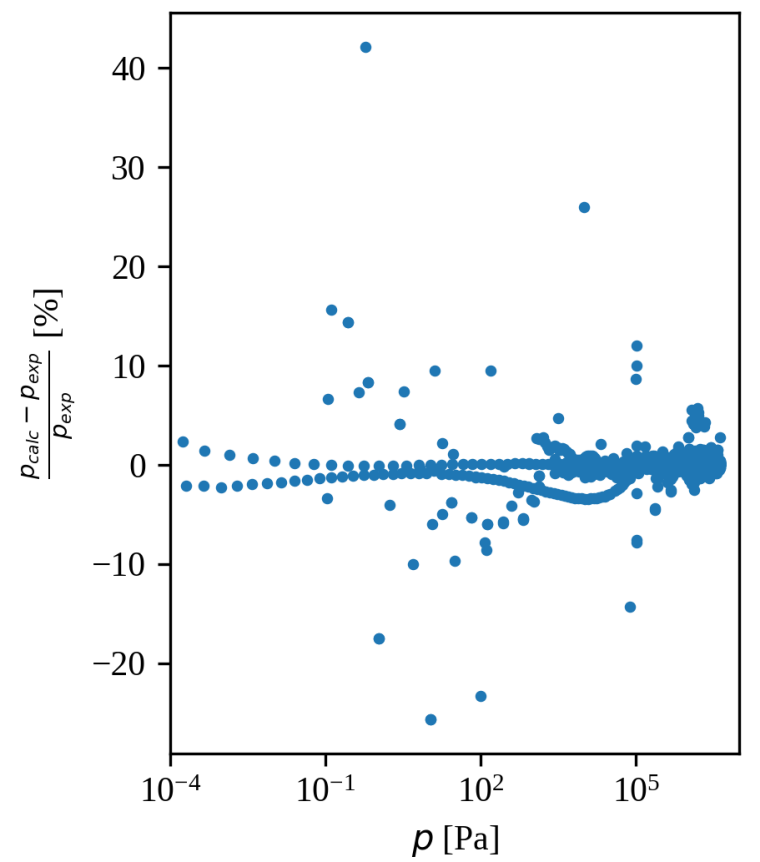

Figure 7: Relative deviations of pressure estimates of propane

sured pressure at low pressure levels as shown in Fig. 7

As the pressure drops, the relative deviations between the estimated and measured pressure ${ }^{1369}$ scatter. At high pressure, the relative devi- ${ }_{1370}$ ations concentrate between $+10 \%$ and $-10 \%$. However, as lower pressure, the distribution of ${ }^{1371}$ the relative deviations widens to between $+40^{1372}$ $\%$ and $-30 \%$. The large scattering of the rela- ${ }^{1373}$ tive deviation of saturation pressure at low lev- ${ }^{1374}$ els results in the increase of relative uncertain- ${ }^{1375}$ ties of saturation pressure with a drop of pres ${ }^{1376}$ sure.

To have a more comprehensive understand- ${ }^{1378}$ ing, the relative uncertainty of pressure in the $\mathrm{e}^{1379}$ single phase region is also studied by the con- ${ }^{1380}$ tour plot of its relative uncertainty over $\mathrm{a}^{1381}$ temperature-specific-volume $(T-1 / \rho)$ plot. The ${ }^{1382}$ contour plot is shown in Fig. 8.

Figure 8 shows that the relative uncertainties ${ }^{1384}$ of pressure of vapor in the single-phase region ${ }^{1385}$ are much less than the relative uncertainties of ${ }^{1386}$ saturation pressure. There are some relative ${ }^{1387}$ uncertainties of pressure of subcooled liquid at ${ }^{1388}$ high densities that are high, but it fits the gen ${ }^{1389}$ eral understanding that the derivative of pres- ${ }^{1390}$ sure with density is high in that regime and $d^{1391}$

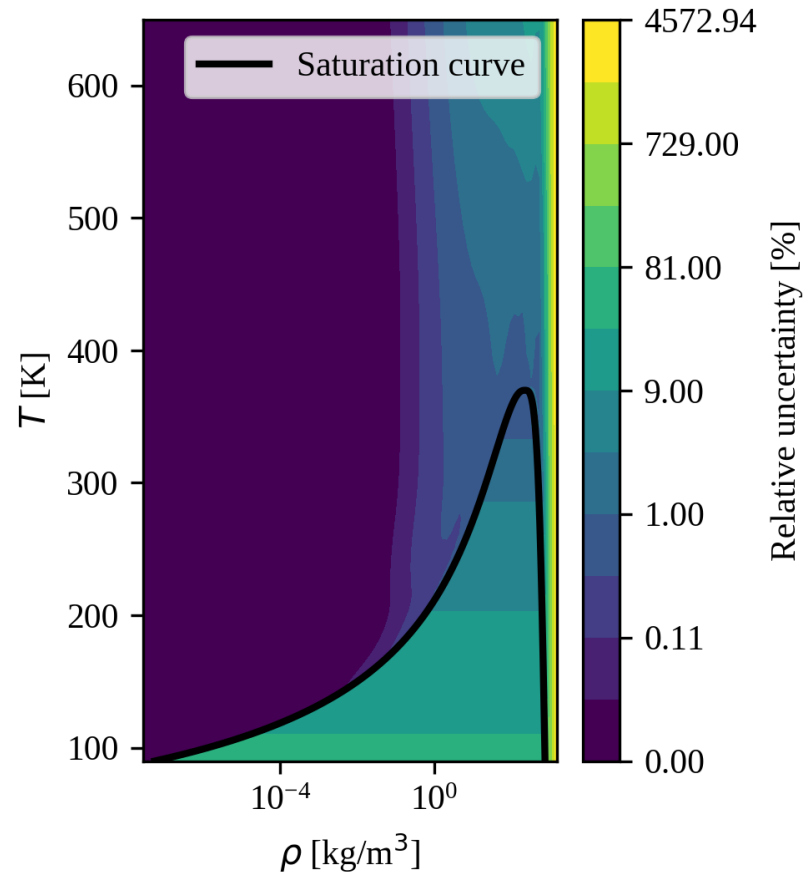

Figure 8: Relative uncertainty of pressure of propane in a $T-1 / \rho$ diagram

hence the relative uncertainty of pressure in the regime is high.

\subsection{Uncertainty of properties in the single-phase region}

To further understand how the uncertainties of EOS change in the single-phase region, the relative uncertainties of speed of sound, isochoric specific heat capacity, isobaric specific heat capacity and density are calculated at different temperature and pressure state points, and the results are shown in Figs. 9a to 9d.

Figure 9 a shows that the relative uncertainty of speed of sound is lower than $4 \%$ in most cases except conditions near the critical point and at pressure higher than $10^{8} \mathrm{~Pa}$. At pressure higher than $10^{8} \mathrm{~Pa}$, only speed of sound data were only collected near $240 \mathrm{~K}$ and density data were collected between $370 \mathrm{~K}$ and $610 \mathrm{~K}$. Other property data were not collected in the pressure regime. Thus the figure only shows an uncertainty value lower than $8 \%$ between $240 \mathrm{~K}$ and $610 \mathrm{~K}$ at pressure higher than $10^{8} \mathrm{~Pa}$, and the uncertainty of the speed of sound remains high at all other temperature values in the pressure regime. 


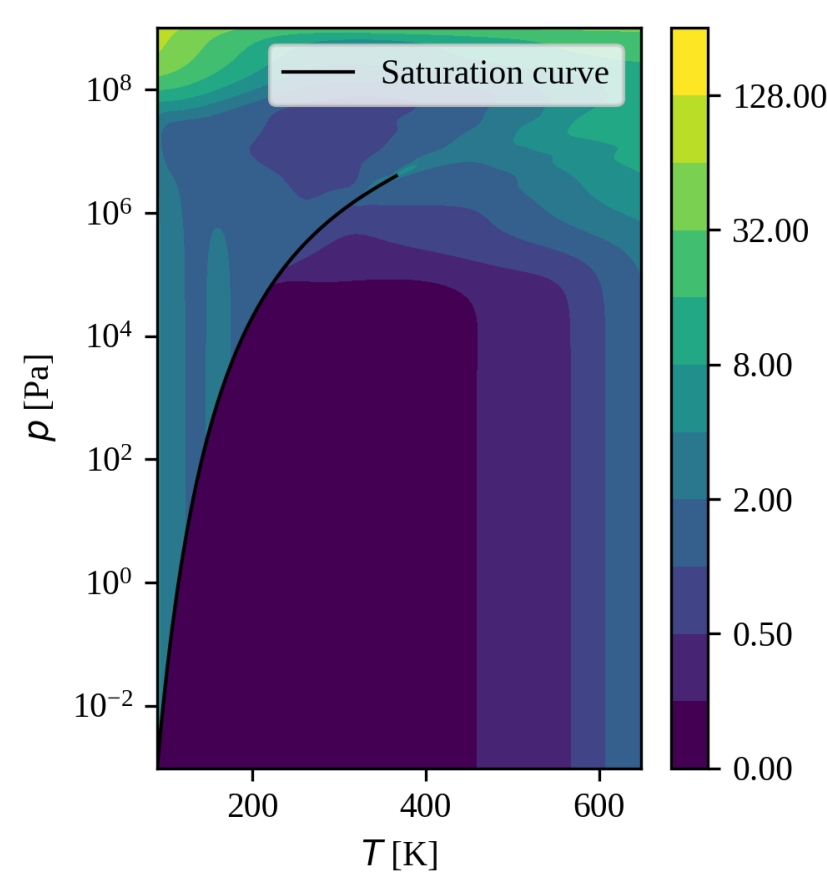

(a) Relative uncertainty of speed of sound

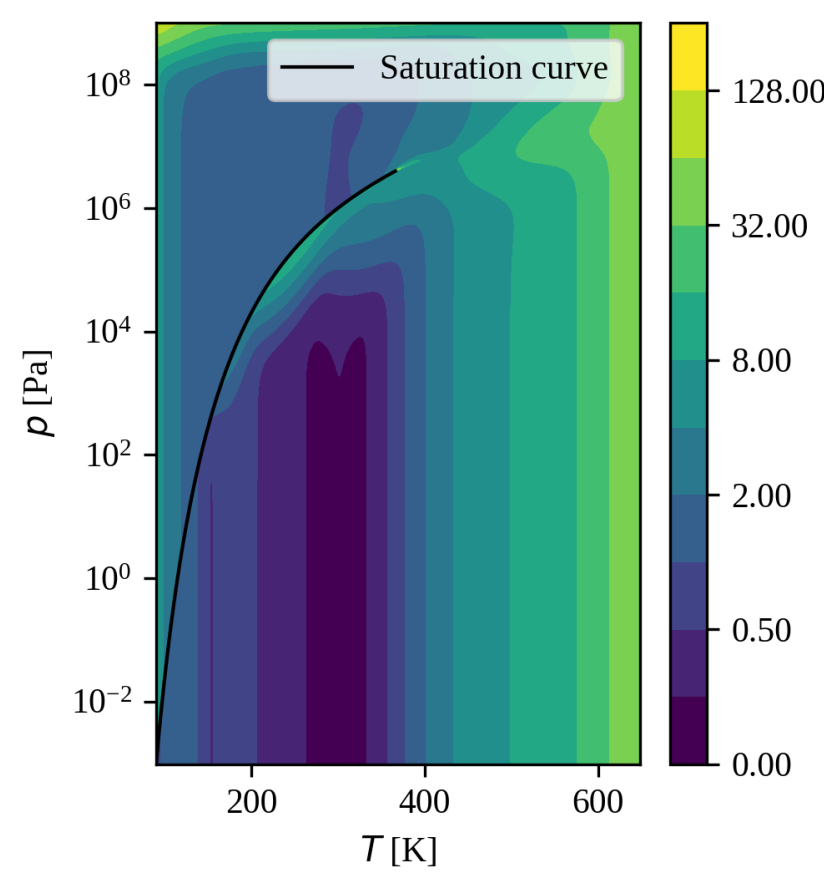

(c) Relative uncertainty of $c_{p}$

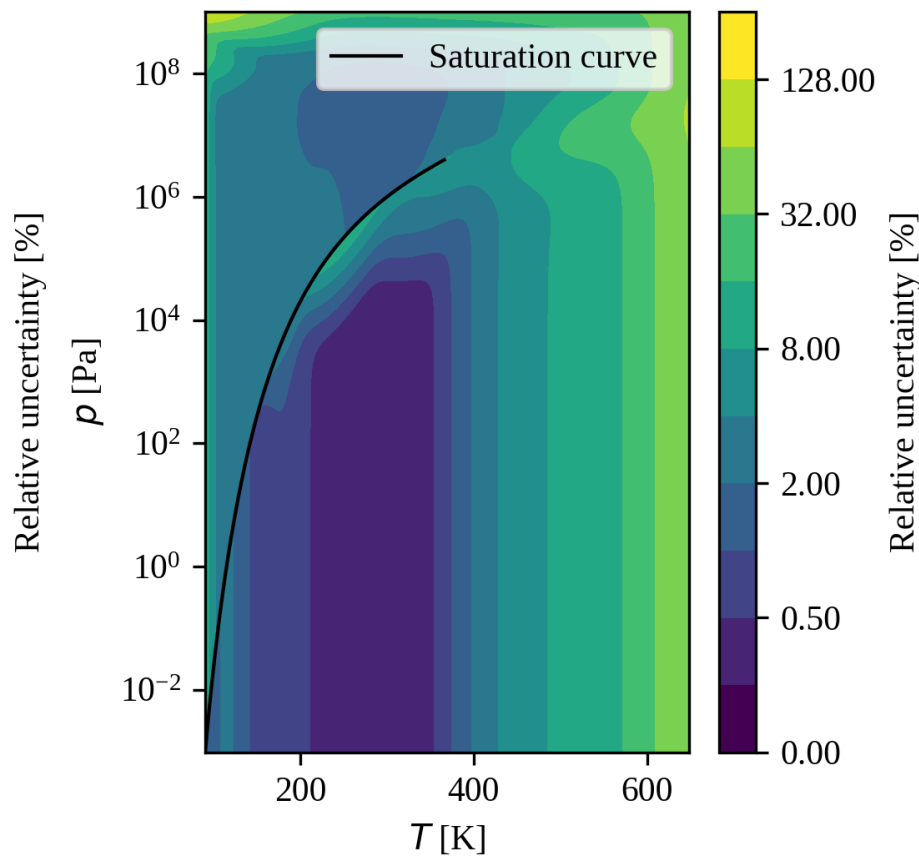

(b) Relative uncertainty of $c_{v}$

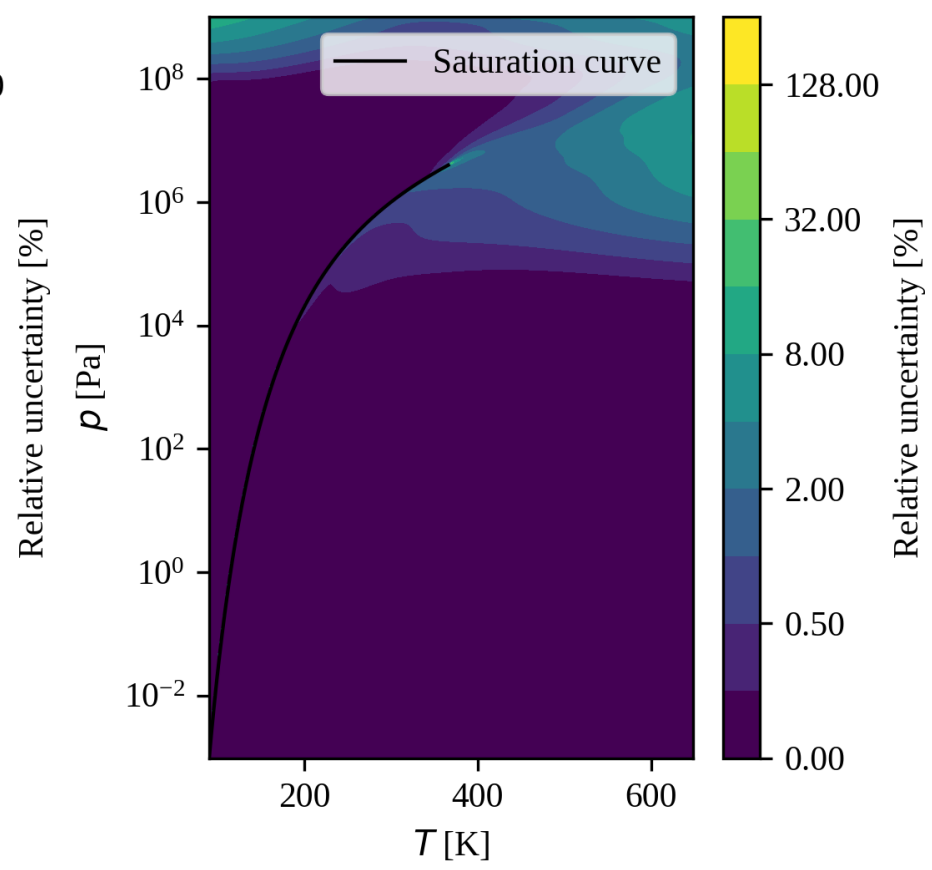

(d) Relative uncertainty of density

Figure 9: Relative uncertainties of propane properties in $p$ - $T$ coordinates

Figures $9 \mathrm{~b}$ and $9 \mathrm{c}$ show a similar pattern in 1399 which the relative uncertainty remains lower ${ }_{1400}$ than $4 \%$ in the subcooled liquid region $\operatorname{and}_{1401}$ highly superheated vapor region. However, the ${ }_{1402}$ relative uncertainties of the specific heat are ${ }_{1403}$ much more significant near the the saturated ${ }_{1404}$ vapor line at $250 \mathrm{~K}$. While the $c_{p}$ and $c_{v}$ of su-1405 perheated vapor near the saturated curve at low temperatures should behave as an ideal gas, the relative uncertainties of $c_{p}$ and $c_{v}$ near the saturated curve at around $250 \mathrm{~K}$ are much higher than their ideal gas counterparts at lower pressure. While this may be caused by the approximation to use model deviation instead of mea- 
surement uncertainty to conduct the calcula-1452 tion, it may also caused by potential overffiting 1453 issues in the part of the EOS related to the ${ }_{1454}$ residual Helmholtz energy.

Overfitting arises when a regression equation ${ }_{1456}$ contains too many coefficients and is fit for ${ }_{1457}$ the random variation in the experimental data ${ }_{1458}$ rather than the systematic relationship between ${ }_{1459}$ variables. Equations with overfitting issues usu-1460 ally result in very accurate prediction at the ex-1461 perimental data points but large model uncer-1462 tainties. ${ }^{40}$ Since $c_{p}$ and $c_{v}$ in the vapor phase ${ }_{1463}$ with the exception of ones near the critical ${ }_{1464}$ point should not differ too much from their ideal ${ }_{1465}$ gas counterparts and the EOS part of the ideal ${ }_{1466}$ gas contribution $\left(\alpha^{0}\right.$ in Eq. (14)) is not complex ${ }_{1467}$ enough to result in significant overfitting, the ${ }_{1468}$ large uncertainty is likely caused by the over-1469 fitting of the residual part of the Helmholtz1470 energy arising from the intermolecular forces 1471 $\left(\alpha^{\mathrm{r}}\right.$ in Eq. (14) $)$. To mitigate these issues and ${ }_{1472}$ the uncertainties, simplification of EOS or con-1473 straints to the coefficients such as penalization ${ }_{1474}$ should be made to reduce these unexpectedly ${ }_{1475}$ high uncertainties. 11

1476

Figure 9d shows a different pattern. Rela-1477 tive uncertainty of density is lower than $0.25 \% 1478$ for all subcooled liquid states and is lower than ${ }_{1479}$ $1 \%$ for most highly superheated vapor states.1480 The relative uncertainty of density of the su-1481 perheated vapor at higher pressures also ranges between $1 \%$ and $4 \%$. The relative uncertainty ${ }^{1482}$ only exceeds $8 \%$ in the critical region.

In summary, the relative uncertainties of $\operatorname{EOS}^{1484}$ are in general large in the critical region. For ${ }^{1485}$ pressure and speed of sound, their relative un- ${ }^{1486}$ certainties in the subcooled liquid region $\operatorname{are}^{1487}$ higher than that of the superheated vapor re- ${ }^{1488}$ gion. For specific heat capacities and densities, ${ }^{1489}$ the relative uncertainties of superheated vapor ${ }^{1490}$ close to saturation are higher than those of the ${ }_{1491}$ subcooled liquid and more highly superheated ${ }_{1492}$ vapor.

\section{Conclusion}

In this paper, a method to calculate the ${ }^{1497}$ uncertainty of thermodynamic properties of ${ }_{1499}^{1498}$
Helmholtz-energy-explicit EOS is developed based on the parameter covariance matrix of nonlinear regression models. The covariance matrix can be calculated from the experimental data in the literature and the Jacobian matrix of the EOS with respect to the parameters of the EOS. To ensure that the effects of the Maxwell criteria and uncertainties of the differences of properties such as enthalpy values are properly calculated, the uncertainty calculation method also involves the linearization of the EOS and the covariance of the differences of properties.

To test the applicability and validity of the calculation method, it is demonstrated by a calculation of the uncertainties of the EOS of propane. The method also enables an analysis of the parameter correlation matrix which shows how the parameters are correlated with each other. It also demonstrates how the use of the Maxwell criteria and the rapid change of properties with respect to the state of the substances around their critical point result in larger uncertainties of properties along the superheated vapor line than the uncertainties of properties in the single-phase region. The results of this study allow users to take into account the uncertainties of the EOS in process model simulations. However, the results also show some limitations of the method:

- The uncertainty analysis does not present the effect of experimental uncertainty of the training data to the EOS because of the lack of information in some data sets of propane. The authors strongly encourage future research to take into account measurement uncertainties, if full measurement uncertainties have been obtained for each data point.

- The uncertainty analysis follows a linear propagation of error approach. This method is the only computationally tractable method that could be used for a nonlinear model like the equation of state studied here. Other more advanced sampling techniques (Markov Chain Monte Carlo (MCMC) sampling methods, etc.) are too computationally expensive to be 
practically applied in technical applica-1545 tions.

- The method assumes a Gaussian distri- ${ }^{154}$ bution for the uncertainties of the EOS, and may fail to calculate the appropri- ${ }_{1550}$ ate value at conditions which uncertain- ${ }_{1551}$ ties distribute differently from the Gaus- ${ }_{1552}$ sian distribution.

- The method assumes that all experimen- ${ }^{1554}$ tal data points are not correlated with ${ }^{1555}$ each other. However, some training data ${ }^{1556}$ points are not associated with compre- ${ }^{1557}$ hensive information on their measure- ${ }^{1558}$ ment uncertainties and hence information ${ }^{1559}$ on correlation between data, and more re- ${ }^{1560}$ search is needed before the effect of corre- ${ }^{1561}$ lation can be comprehensively accounted ${ }^{1562}$ for by an uncertainty calculation method. ${ }^{1563}$

- The method does not involve the struc-1565 tural uncertainty in Table 1 .

- The state-of-the-art fitting process of the ${ }_{1568}$ EOS includes addition and removal of ${ }_{1569}$ data points and constraints in an itera- ${ }_{1570}$ tive fashion, and it is computationally expensive to involve the constantly changing and exact objective function for the ${ }_{1571}$ fit of the EOS into the uncertainty calculation method. Thus the method has to ${ }^{1572}$ use an approximated objective function of ${ }^{1573}$ the EOS for the uncertainty calculation, ${ }^{1574}$ and it is unknown how much error is in-1575 troduced by the difference as a result. $\quad{ }^{1576}$ 1577

Last but not least, it is recommended that users and developers of EOS perform this type ${ }^{1578}$ of analysis in order to obtain insights about the ${ }^{1579}$ uncertainties of the properties calculated by the ${ }^{1580}$ EOS. Since the method does not incorporate ${ }^{1581}$ other sources of uncertainties such as experi- ${ }_{1582}$ mental uncertainties shown in Table 1, other ${ }_{1583}$ methods to involve the experimental uncertain- ${ }_{1584}$ ties should also be used to have a comprehensive ${ }_{1585}$ understanding of uncertainties of EOS.

Supporting Information. Supporting Information is provided in three files. One file ${ }_{1587}$ contains the mathematical derivation related to1588 the differences of the expressions in this paper from that in some statistical textbooks, derivation for the Gibbs energy approximation in Fig. 1, the parameter identifiability analysis results, the Python code used to calculate the uncertainties of the thermodynamic properties of propane and the list of sources of experimental data being used in the study. The covariance matrix is provided in pcov.csv, and the correlation matrix is provided in pcor.csv. A Jupyter Notebook and the associated Python code and data files are also provided in a separate zip file jupyter-notebook-demo.zip to illustrate the use of the code.

Acknowledgement. The authors would like to acknowledge Allan H. Harvey at National Institute of Standards and Technology, Boulder, CO, USA for his comments on the manuscript draft. Commercial equipment, instruments, or materials are identified only in order to adequately specify certain procedures. In no case does such identification imply recommendation or endorsement by the National Institute of Standards and Technology, nor does it imply that the products identified are necessarily the best available for the purpose.

\section{References}

(1) Frutiger, J.; Andreasen, J.; Liu, W.; Spliethoff, H.; Haglind, F.; Abildskov, J.; Sin, G. Working fluid selection for organic Rankine cycles Impact of uncertainty of fluid properties. Energy 2016, 109, 987997.

(2) Cheung, H.; Braun, J. E. A general method for calculating the uncertainty of virtual sensors for packaged air conditioners. Int. J. Refrig 2016, 63, 225-236.

(3) Huang, G.; Wang, S.; Xu, X. A robust model predictive control strategy for improving the control performance of air-conditioning systems. Energy Convers. Manage. 2009, 50, 2650-2658.

(4) Shan, K.; Wang, S.; Xiao, F.; Sun, Y. Sensitivity and uncertainty analysis of cool- 
ing water control strategies. HVACER Re-1633 search 2013, 19, 435-443.

1634

(5) Reddy, T. A.; Niebur, D.; Ander-1635 sen, K. K.; Pericolo, P. P.; Cabrera, G.1636 Evaluation of the Suitability of Differ-1637 ent Chiller Performance Models for On-1638 Line Training Applied to Automated Fault Detection and Diagnosis (RP-1139). ${ }^{1639}$ HVACER Research 2003, 9, 385-414.

(6) Proppe, J.; Reiher, M. Reliable estima-1642 tion of prediction uncertainty for physico-1643 chemical property models. J. Chem. Theory Comput. 2017, 13, 3297-3317. 1645

(7) Hukkerikar, A.; Sarup, B.; Ten Kate, A.;1646 Abildskov, J.; Sin, G.; Gani, R. Group- ${ }^{1647}$ contribution $+(\mathrm{GC}+)$ based estimation of properties of pure components: Improved ${ }_{1649}^{1648}$ property estimation and uncertainty anal- ${ }_{1650}$ ysis. Fluid Phase Equilib. 2012, 321, 25- ${ }^{-1650}$ 43.

(8) Morgan, J.; Bhattacharyya, D.; Tong, C.; ${ }_{1653}^{1652}$ Miller, D. C. Uncertainty quantification of ${ }_{1654}$ property models: Methodology and its ap- ${ }_{1655}$ plication to CO2-loaded aqueous MEA solutions. AIChE J. 2017, 61, 1822-1839. ${ }^{1656}$

(9) Kamei, A. Beyerlein, S. W . J Jacob- ${ }^{1657}$ sen, R. T. Application of nonlinear regres- ${ }_{1659}$ sion in the development of a wide range ${ }_{1660}$ formulation for HCFC-22. Int. J. Thermo- ${ }_{1661}$ phys. 1995, 16, 1155-1164.

1662

(10) Wagner, W.; Pruß, A. The IAPWS For ${ }_{1663}$ mulation 1995 for the Thermodynamic ${ }_{1664}$ Properties of Ordinary Water Substance $_{1665}$ for General and Scientific Use. J. Phys. ${ }_{1666}$ Chem. Ref. Data 2002, 31, 387-535.

1667

(11) Lemmon, E. W.; Jacobsen, R. T. Equa- ${ }_{1668}$ tions of State for Mixtures of R-32, R-1669 125, R-134a, R-143a, and R-152a. J. Phys.1670 Chem. Ref. Data 2004, 33, 593.

1671

(12) Lemmon, E. W.; McLinden, M. O.; Wag-1672 ner, W. Thermodynamic Properties of 1673 Propane. III. A Reference Equation of ${ }_{1674}$ State for Temperatures from the Melt-1675 ing Line to $650 \mathrm{~K}$ and Pressures up to1676
1000 MPa. J. Chem. Eng. Data 2009, 54, 3141-3180.

(13) Lemmon, E. W. Pseudo-Pure Fluid Equations of State for the Refrigerant Blends R-410A, R-404A, R-507A, and R-407C. Int. J. Thermophys. 2003, 24, 991-1006.

(14) Cheung, H.; Bach, C. K. Prediction of uncertainty of 10-coefficient compressor maps for extreme operating conditions. IOP Conference Series: Materials Science and Engineering. 2015; p 012078.

(15) ASME, ASME PTC 19.1-2013 Test Uncertainty Performance Test Codes; The American Society of Mechanical Engineers: New York, 2013.

(16) JCGM, JCGM 100:2008 Evaluation of measurement data Guide to the expression of uncertainty in measurement; Joint Committee for Guides in Metrology, 2008.

(17) ASME, ASME VEV 10-2006 Guide for Verification and Validation in Computational Solid Mechanics; American Society of Mechanical Engineers: New York, 2006.

(18) Cheung, H.; Wang, S. A comparison of the effect of empirical and physical modeling approaches to extrapolation capability of compressor models by uncertainty analysis: A case study with common semiempirical compressor mass flow rate models. Int. J. Refrig 2018, 86, 331-343.

(19) Feistel, R.; Lovell-Smith, J. W.; Saunders, P.; Seitz, S. Uncertainty of empirical correlation equations. Metrologia 2016, 53, 1079 .

(20) Lovell-Smith, J. W.; Saunders, P.; Feistel, R. Unleashing Empirical Equations with Nonlinear Fitting and GUM Tree Calculator. Int. J. Thermophys. 2017, 38, 148.

(21) Frutiger, J.; Bell, I.; O'Connell, J. P.; Kroenlein, K.; Abildskov, J.; Sin, G. Uncertainty assessment of equations of state with application to an organic Rankine cycle. Mol. Phys. 2017, 115, 1225-1244. 
(22) Lötgering-Lin, O.; Schöniger, A.;1720 Nowak, W.; Gross, J. Bayesian Model 1721 Selection Helps To Choose Objectively ${ }_{1722}$ between Thermodynamic Models: A Demonstration of Selecting a Viscosity ${ }^{1723}$ Model Based on Entropy Scaling. Ind. ${ }^{1724}$ Eng. Chem. Res. 2016, 55, 10191-10207. ${ }^{1725}$

(23) Lasala, S.; Chiesa, P.; Privat, R. ${ }^{1726}$ Jaubert, J.-N. Modeling the Thermody- ${ }^{1727}$ namics of Fluids Treated by CO2 Capture ${ }^{1728}$ Processes with PengRobinson + Residual ${ }^{1729}$ Helmholtz Energy-Based Mixing Rules. ${ }^{1730}$ Industrial \&5 Engineering Chemistry Re- ${ }^{1731}$ search 2017, 56, 2259-2276.

(24) Kline, S. J.; McClintock, F. A. Describ- ${ }^{1733}$ ing uncertainties in single-sample experi- ${ }^{1734}$ ments. Mech. Eng. 1953, 75, 3-8.

(25) Sin, G.; Gernaey, K. V.; Lantz, A. E. Good 1737 modeling practice for PAT applications: Propagation of input uncertainty and sen- ${ }^{1738}$ sitivity analysis. Biotechnol. Progr. 2009, ${ }^{1739}$ 25, 1043-1053.

(26) Dong, Q.; Chirico, R. D.; Yan, X.; Hong, X.; Frenkel, M. Uncertainty Re- ${ }^{1742}$ porting for Experimental Thermodynamic ${ }^{1743}$ Properties. J. Chem. Eng. Data 2005, 50, ${ }_{1745}^{174}$ 546-550.

(27) Seber, G.; Wild, C. J. Nonlinear regres- ${ }^{1746}$ sion; John Wiley \& Sons, Inc.: Hoboken, ${ }^{1747}$ NJ, USA, 1989.

(28) Sin, G.; Gernaey, K. V.; Neumann, M. B.;1750 van Loosdrecht, M. C. M.; Gujer, W. ${ }^{1751}$ Global sensitivity analysis in wastewa- ${ }^{1752}$ ter treatment plant model applications: ${ }^{1753}$ Prioritizing sources of uncertainty. Water Res. 2011, 45, 639-651.

1755

(29) Frutiger, J.; Marcarie, C.; Abildskov, J.,1756 Sin, G. A Comprehensive Methodology for ${ }^{1757}$ Development, Parameter Estimation, and ${ }^{1758}$ Uncertainty Analysis of Group Contribu- ${ }^{1759}$ tion Based Property ModelsAn Application to the Heat of Combustion. J. Chem. ${ }_{1761}$ Eng. Data 2016, 61, 602-613.

(35) Thorade, M.; Saadat, A. Partial derivatives of thermodynamic state properties for dynamic simulation. Env. Earth Sci. 2013, 70, 3497-3503.

(36) Diky, V.; Chirico, R. D.; Muzny, C. D.; Kazakov, A. F.; Kroenlein, K.; Magee, J. W.; Abdulagatov, I.; Kang, J. W.; Frenkel, M. ThermoData Engine (TDE) software implementation of the dynamic data evaluation concept. 7. Ternary mixtures. J. Chem. Inf. Model. 2011, 52, 260-276.

(37) Frenkel, M.; Chirico, R. D.; Diky, V.; Yan, X.; Dong, Q.; Muzny, C. ThermoData Engine (TDE): Software Implementation of the Dynamic Data Evaluation Concept. J. Chem. Inf. Model. 2005, 45, 816-838.

(38) Diky, V.; Chirico, R. D.; Muzny, C. D.; Kazakov, A. F.; Kroenlein, K.; Magee, J. W.; Abdulagatov, I.; Kang, J. W.; Gani, R.; Frenkel, M. 
ThermoData Engine (TDE): Software implementation of the dynamic data evaluation concept. 8. Properties of material streams and solvent design. J. Chem. Inf. Model. 2012, 53, 249-266.

(39) Diky, V.; Chirico, R. D.; Muzny, C. D.; Kazakov, A. F.; Kroenlein, K.; Magee, J. W.; Abdulagatov, I.; Frenkel, M. ThermoData Engine (TDE): Software implementation of the dynamic data evaluation concept. 9. Extensible thermodynamic constraints for pure compounds and new model developments. J. Chem. Inf. Model. 2013, 53, 3418-3430.

(40) Hawkins, D. M. The Problem of Overfitting. J. Chem. Inf. Comput. Sci. 2004, 44, $1-12$.

(41) Babyak, M. A. What You See May Not Be What You Get: A Brief, Nontechnical Introduction to Overfitting in RegressionType Models. Psychosom. Med. 2004, 66, 411-421. 